\title{
An LES Investigation of the Near-Wake Flow Topology of a Simplified Heavy Vehicle
}

\author{
Anirudh N. $\operatorname{Rao}^{1}$ (D) . Jie Zhang ${ }^{1}$ (D) . \\ Guglielmo Minelli $^{1}$ (D) Branislav Basara ${ }^{2}$. \\ Siniša Krajnović ${ }^{1}$ (D)
}

Received: 12 January 2018 / Accepted: 22 June 2018 / Published online: 25 September 2018

(C) The Author(s) 2018

\begin{abstract}
Recent experimental investigations by McArthur et al. (J. Fluids Struct. 66, 293$314,2016)$ in the wake of a simplified heavy vehicle or commonly known as the ground transportation system (GTS) model has shown that the flow topology is invariant over a large range of Reynolds numbers $\left[2.7 \times 10^{4}-2 \times 10^{6}\right]$. Numerical simulations are performed to investigate the initial flow topology at a Reynolds number of $2.7 \times 10^{4}$, using well-resolved large eddy simulations (LES). In the vertical midplane behind the GTS, a flow state which is anti-symmetric to that reported in McArthur et al. (J. Fluids Struct. 66, 293-314, 2016) is observed here, thereby, confirming the possibility of occurrence of the complementary bi-stable flow state. The occurrence of this bi-stable state does not depend on the ground clearance between the GTS and the ground plane, as a similar flow topology is observed at both small and large gap heights. Furthermore, the flow topology in the vertical midplane is also found to be insensitive to the incoming flow for small yaw angles. However, complex flow behaviour is observed in the wake for larger yaw angles, where the flow topology in the vertical midplane becomes nearly symmetric, while an asymmetric flow topology is now observed in the lateral midplane in the near wake. Furthermore, the corner vortices which originate from either side at the front of the model merge in the far wake, leading to a large vortex structure nearly equal to the height of the model. The near-wake topology of the GTS is analysed and compared with previous studies for a range of scenarios, and the forces on the GTS are computed.
\end{abstract}

Keywords Wakes $\cdot$ Bi-stability $\cdot$ LES $\cdot$ Heavy vehicles

Anirudh N. Rao

anirudh.rao@chalmers.se

Siniša Krajnović

sinisa.krajnovic@chalmers.se

1 Department of Mechanics and Maritime Sciences, Chalmers University of Technology, Göteborg, 41296, Sweden

2 AVL List GmbH, Advanced Simulation Technologies, Hans-List-Platz 1, 8020, Graz, Austria 


\section{Introduction}

The ground transportation system (GTS) model is a simplified cab-over-engine truck model and is representative of a tractor-trailer combination without any intermediate gap. The earliest experimental investigations of the GTS model by [10] and [71] were performed with the aim of reducing drag by the use of active and passive flow control devices (several addon devices such as slanted rear end and boat-tails to the base of the model) ([4, 8, 9, 14, $28,44,47,54]$, and others). Storms et al. [71] reported a $19 \%$ reduction in the drag coefficient by the use of boat-tail plates. To accurately compare the flow features in the near wake with the experimental studies, several numerical investigations were performed by the use of computational fluid dynamics (CFD). Roy et al. [63] obtained steady state solutions at $R e=2 \times 10^{6}$ using Reynolds-averaged Navier-Stokes (RANS) $k-\omega$ Menter model and Spalart-Allmaras (SA) model. They assessed the capability of these model by comparing the flow structures and surface pressure data obtained from the work of [71]. Behind the base of the model, two symmetrical vortices were observed in the numerical computations, while the experimental particle image velocimetry (PIV) data showed an asymmetrical vortex structure, with a bottom vortex closer to the base and the top vortex at a distance of a trailer-width downstream of the base. In the horizontal midplane, two symmetrical counterrotating vortices were captured in both the experiments and numerical simulations. While the numerical simulations did not accurately predict the pressure coefficient on the base, the prediction of the drag coefficient from the $k-\omega$ Menter model was in good agreement with the experiments. A similar investigation by [65] using RANS models failed to accurately capture the asymmetrical flow field in the vertical midplane of the model, although the Wilcox $1988 k-\omega$ model showed a larger degree of asymmetry as compared to the SA and the Mentor SST models.

Ortega et al. [49] performed LES on a truncated GTS model, simulating the flow around the rear of the body which extends to a distance equal to the height of the model from the base. The simulations performed on a coarse and a fine mesh predicted the formation of an asymmetrical vortex structure in the near wake. The mean flow topology in the vertical midplane on both meshes predicted a large triangular-shaped vortex at the top and closer to the base, and the smaller vortex at the bottom further away from the base. They also observed the formation of two counter-rotating vortices from the top corners of the model which extend far downstream in the wake.

Unaune et al. [73] performed numerical simulations using detached eddy simulations (DES) and RNG (Re-Normalisation Group) $k-\varepsilon$ turbulence model at $R e=2 \times 10^{6}$ for the GTS model. The transient vortical structures and the shedding behind the body are captured by their DES, while the URANS (unsteady RANS) predictions approached a steady state result. The resulting flow topology observed in their simulations is opposite to that observed in [45] and similar to the LES of [49]; although the vortex structures observed are elongated in the streamwise direction. They further indicate that enough "upwash" was not generated to produce vortical structures similar to that observed in [71]. Nonetheless, the predicted drag coefficient from their URANS and DES were within $0.24 \%$ and $1.25 \%$ of the experimentally observed values, respectively. Numerical simulations were performed by [17] using body-fitted and immersed boundary grids to investigate the flow past a GTS at $R e=2 \times 10^{6}$. Using RANS $k-\varepsilon$ model, they predicted the flow fields and the drag coefficient for the GTS. Symmetric vortices were observed in the vertical midplane, and the two grids over-predicted the drag coefficient of the GTS as compared to [71]. Gunes [23] observed a flow topology similar to the experimental study of [71] using the RNG $k-\varepsilon$ turbulence model on an unstructured grid, with a harmonic motion of the upper and the lower 
vortices at rear of the GTS, which subsequently influenced the pressure coefficient on the base and the drag coefficient of the GTS.

More recently, [45] performed experimental investigations on a 1:40 scale GTS model in a water channel. The flow topology observed in the vertical midplane in [45] at $R e \simeq$ $2.7 \times 10^{4}$ and [71] at $R e=2 \times 10^{6}$ was similar, given the large disparity in Reynolds numbers. McArthur et al. [45] also investigated the influence of the gap height on the wake topology. As the gap height between the ground and the model is increased from $0.03 \mathrm{H}$ to $0.14 H$ (where $H$ is the height of the GTS model), an asymmetrical flow topology was observed, with the size of the lower vortex increasing with gap height. However, for gap heights in excess of $0.14 \mathrm{H}$, the flow topology remained unaltered, with minimal change in the location of the centres of the vortices. A more recent study by [6] classified the wake topology in the vertical midplane into four categories based on the ratio of the underbody velocity to the freestream velocity for a simplified truck model. The flow topology such as the one observed in the vertical midplane of the GTS model for $G / H \geqslant 0.14$ occurred when this ratio was $\gtrsim 0.5$. The flow topology in the vertical midplane of the GTS predicted by numerical simulations of [49] and [73] appear to be anti-symmetrical to that predicted by [45] and [71]. The height-to-width ratio of the GTS model is 1.392, while the width-toheight ratio of the squareback Ahmed body is 1.35 , where bi-stability (which refers to the switching from one reflectional symmetry breaking flow state to another) has been previously reported ([16, 19-21, 42, 76] and others); indicating that bi-stable phenomenon could indeed be plausible in the vertical midplane of the GTS [45].

While the GTS model is a simplified version of the actual trucks used today, the flow topology at the rear of the trailer is invariant to the complicated geometrical features such as mirrors, wheels and side skirts [12, 40]. In this study, we use large eddy simulations (LES) to predict the near-wake topology of a simplified GTS model at $R e \simeq 2.7 \times 10^{4}$ in order to compare directly with the experimental observations of [45]. The goals of this study are: to validate the LES for when the incoming flow is at zero yaw angle, and to detail the nearand far-wake flow topologies at larger yaw angles, with the latter not having received sufficient attention in previous studies. The results from this investigation are also intended to serve as a baseline for future comparison with other turbulence modelling approaches. The remainder of the article is organised as follows: Section 2.1 elucidates the problem setup, while Section 2.2 briefly details the numerical method employed. The results from LES for yaw angles of $\beta=0^{\circ}$ is detailed in Section 3.1, where the flow topology observed is antisymmetric to that observed in [45]. A qualitative comparison describing this complimentary flow state follows. The flow topology is further investigated for non-zero yaw angles; first, to investigate if a small asymmetry brought about by the incoming flow at an yaw angle of $\beta=2.5^{\circ}$ is capable of obtaining a flow state similar to previous experimental studies, and second, to describe the complex flow dynamics at $\beta=10^{\circ}$. The results from these simulations are presented in Section 3.2, and this is followed by conclusions in Section 4.

\section{Methodology}

\subsection{Problem setup}

The GTS model, first proposed by [10], is characteristic of a bluff body; with an ellipticalshaped front nose, and an elongated flat section with a squareback base at the rear. The side, top and bottom edges at the front are curved, and is representative of a truck cabin with no gap to the trailer. In this investigation, the curved A-pillars at the front of the model are 


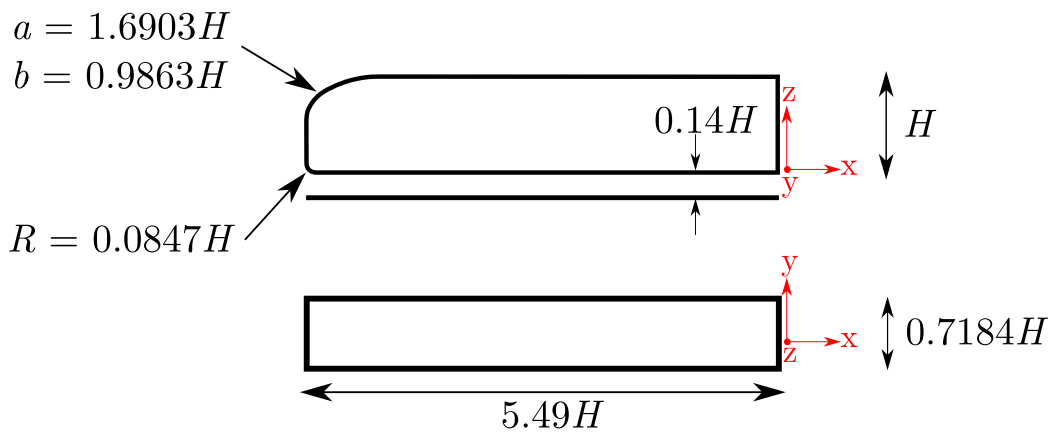

(a)

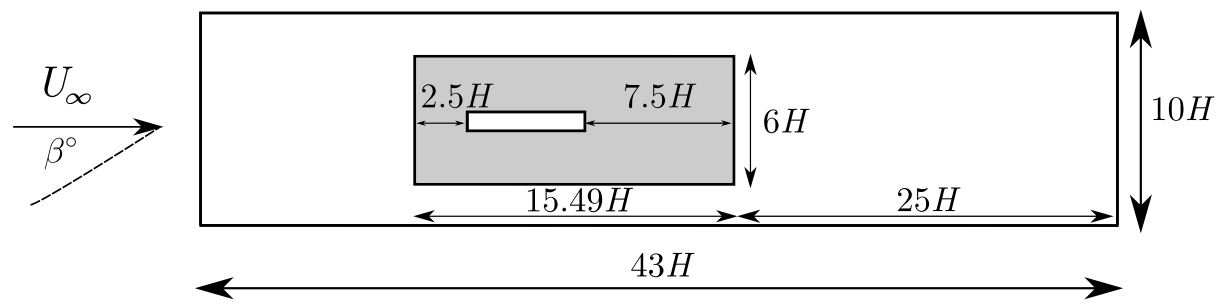

(b)

Fig. 1 a Schematic of the GTS model showing the major dimensions of the model. The upper frontal shape of the model is elliptical, with the dimensions of the semi-major axis $(a)$ and semi-minor axis $(b)$ shown. The bottom edge at the front of the model is filleted with a radius, $R$. b Schematic of the computational domain in plan view, with the no-slip region shaded in gray. The dimensions of the GTS model and the computational domain are normalised by the height of the GTS

removed, resulting in a sharp frontal edge along the side in order to aid the construction of a purely hexahedral mesh. This also ensures a fixed separation point for the flow and may perhaps lead to a larger recirculation region at the sides as compared to that observed in previous experimental studies. Essentially, the two-dimensional profile of the GTS model in the vertical midplane has been extruded to a length equal to its width. The schematic of the GTS model used in this study is shown in Fig. 1a, with the major dimensions being normalised by the height $(H)$ of the model. Two cases are considered in this study; one where the model is placed close to the ground at a height of $0.14 \mathrm{H}$, and the second case where the body is placed at a height of $1.1 \mathrm{H}$ above the ground to study the influence of the shape of the model on the flow topology in the absence of ground effect.

The GTS model is placed at the centre of the computational domain (see Fig. 1b), with the domain boundaries placed at large distances from the model to ensure a blockage of $\lesssim 1 \%$ based on the ratio of the frontal area of the GTS to the inlet area of the domain $((0.32385 m \times 0.4508 m) /(4.508 m \times 4.1203 m) \simeq 0.8 \%)$. The inlet and outlet boundaries are at a distance of $18 \mathrm{H}$ and $25 \mathrm{H}$ from the origin, which is located at the bottom edge of the base of the model on the symmetry plane. The lateral and top boundaries extend $5 \mathrm{H}$ and $9 \mathrm{H}$ from the origin, respectively, and are assigned symmetry boundary conditions when the zero yaw angle cases. The ground boundary condition used here is set to replicate the experimental work of [45], with a slip wall enclosing a no-slip wall. The no-slip wall extends $2.5 \mathrm{H}$ and $7.5 \mathrm{H}$ upstream and downstream of the model, respectively, and $3 \mathrm{H}$ on either side of the model in the lateral direction on the ground plane. While experimental investigations usually have cylindrical or streamlined supports, these were not used for the model considered 
here, thereby leading to an unperturbed underbody flow. The inlet boundary condition was assigned a uniform inflow velocity of $U_{\infty}=1.2768 \mathrm{~ms}^{-1}$, leading to a Reynolds number $R e_{W} \simeq 2.7 \times 10^{4}$, based on the width $(W)$ of the model. Here, $R e_{W}=U_{\infty} W / v$ is defined as the ratio of the inertial to the viscous forces, where, $v$ is the kinematic viscosity of the fluid. It may be noted that the width of the GTS has been used as the characteristic length for the Reynolds number and the Strouhal numbers, while other quantities are nondimensionalised by the height of the GTS in accordance with previous studies [45, 71]. The homogeneous Neumann boundary condition is used at the outlet. To investigate the possibility of occurrence of bi-stable flow at non-zero yaw angles [76, 77], the incoming flow is yawed at angles of $\beta=2.5^{\circ}$ and $10^{\circ}$ with respect to the vertical midplane of the GTS as shown in Fig. 1b. For these cases, the lateral and the top boundaries were assigned the same boundary conditions as the inlet. The estimated height of the boundary layer on the no-slip wall at the front of the model (in its absence) is $\simeq 0.12 H$, which is nearly equal to the gap height between the model and the ground [66].

\subsection{Numerical formulation}

The governing LES equations are the incompressible Navier-Stokes and the continuity equations, filtered with the implicit spatial filter of characteristic width, $\Delta=\left(\Delta_{x} \Delta_{y} \Delta_{z}\right)^{1 / 3}$, where $\Delta_{x}, \Delta_{y}$ and $\Delta_{z}$ are the computational cell sizes in the $x, y$ and $z$ coordinate directions, respectively.

$$
\begin{gathered}
\frac{\partial \bar{u}_{i}}{\partial t}+\frac{\partial}{\partial x_{j}}\left(\bar{u}_{i} \bar{u}_{j}\right)=-\frac{1}{\rho} \frac{\partial \bar{p}}{\partial x_{i}}+v \frac{\partial^{2} \bar{u}_{i}}{\partial x_{j} \partial x_{j}}-\frac{\partial \tau_{i j}}{\partial x_{j}} \\
\frac{\partial \bar{u}_{i}}{\partial x_{i}}=0
\end{gathered}
$$

Here, $\bar{u}_{i}$ and $\bar{p}_{i}$ are the resolved velocity and pressure, respectively, and the bar over the variable denotes the operation of filtering. The influence of the small scales in Eq. 1 appears in the subgrid-scale (SGS) stress tensor, $\tau_{i j}=\overline{u_{i} u_{j}}-\bar{u}_{i} \bar{u}_{j}$. The algebraic eddy viscosity model originally proposed by [68] is used in the present work for its simplicity and low computational cost. The standard Smagorinsky model represents the anisotropic part of the SGS stress tensor, $\tau_{i j}$ as

$$
\tau_{i j}-\frac{1}{3} \delta i j \tau_{k k}=-2 v_{s g s} \bar{S}_{i j}
$$

where the SGS viscosity,

$$
v_{s g s}=\left(C_{s} f \Delta\right)^{2}|\bar{S}|
$$

and,

$$
\bar{S}=\sqrt{2 \bar{S}_{i j} \bar{S}_{i j}}
$$

Here, $\bar{S}_{i j}$ is the is the resolved rate-of-strain tensor, and is given by

$$
\bar{S}_{i j}=\frac{1}{2}\left(\frac{\partial \bar{u}_{i}}{\partial \bar{x}_{j}}+\frac{\partial \bar{u}_{j}}{\partial \bar{x}_{i}}\right)
$$

The Smagorinsky model coefficient, $C_{s}=0.1$ was chosen for its simplicity and low computational cost, and has previously used in bluff body flows [30, 32, 55, 56, 69]. Since the resolved strain-rate tensor, $\bar{S}_{i j}$, does not vanish at the wall, the value of the coefficient (usually between $C_{s}=0.1$ and 0.2 [64]), should be adjusted to partially take into account 
the effect of the wall. Thus, the damping function, $f$, is used to damp the turbulence length scales next to the wall. The Van Driest damping function is given by,

$$
f=1-\exp \left(\frac{-n^{+}}{25}\right)
$$

where, $n^{+}$is the wall normal distance in viscous units.

The LES equations are discretised with a commercial finite volume solver, AVL FIRE ${ }^{\mathrm{TM}}$ 2014 [1], to solve the incompressible equations using a collocated grid arrangement, where the equations are solved for the velocities. The convective fluxes are approximated by a blend of $95 \%$ linear interpolation of second-order accuracy (central differencing scheme) and of 5\% upwind differences of first-order accuracy (upwind scheme). The diffusive terms containing viscous plus subgrid terms are approximated by a central differencing interpolation of second-order accuracy. It should be noted that for complex geometries using non-equidistant computational grids a commutation error is introduced, since the formulation of Eq. 1 assumes that the filtering operation and spatial derivation commutes, which is not true on non-equidistant grids. This error is of the same order as the truncation error of the second-order linear interpolation scheme $\left(\Delta^{2}\right)$ (see [18]). The time-marching procedure is done using the implicit second-order accurate three-time level scheme:

$$
\left(\frac{\mathrm{d} \phi}{\mathrm{d} t}\right)_{n}=\frac{3 \phi^{n}-4 \phi^{n-1}+\phi^{n-2}}{2 \Delta t_{n}} ; \Delta t_{n}=t-t_{n-1}=t_{n-1}-t_{n-2}
$$

where, indices "n", "n-1" and "n-2" denote the three time levels. To determine the pressure, the discrete form of the filtered continuity equation (Eq. 2) is converted into an equation for the pressure correction which is solved by using the SIMPLE (Semi-Implicit Method for Pressure Linked Equations) algorithm [57]. The influence of a larger value of central differencing of $98 \%$ blend and pure central differencing (100\%) on the flow topology was also investigated for the convective fluxes on the medium mesh. On increasing the blending factor, the length of the recirculation region in the near wake decreased, with the location of the centre of the vortices closer to the base of the GTS model. This resulted in a larger value of the mean drag coefficient (also see Table 2), although the flow topology remained the same. However, these simulations showed spurious flow structures near the nose of the GTS model, and hence, a central difference blending factor more than 0.95 was not used for the momentum equations (also see [37]). The numerical formulation used here has previously been validated for a wide range of bluff body flows [29, 31, 34-36, 47, 50-53].

\subsection{Computational grid and validation}

Three hexahedral meshes were constructed in Ansys ICEM-CFD grid generation package to investigate the influence of the spatial resolution on the flow topology in the near wake. For the GTS model at a gap height of $G=0.14 H$, the coarse, medium and fine meshes consisted of approximately 3.88, 8.35 and 10.78 million elements, respectively. Although the spatial resolution between the medium mesh and the fine mesh was not significantly higher, the spatial resolution in the streamwise direction in the near-wake region was increased for the fine mesh as compared to the medium mesh, resulting in a mesh with a cell count nearly 1.3 times that of the medium mesh. For the case where the GTS model is at a height of $1.1 \mathrm{H}$ above the ground, the mesh consisted of approximately 9.18 million elements, with the distribution of elements similar to the medium mesh. Shown in Fig. 2a is the surface 


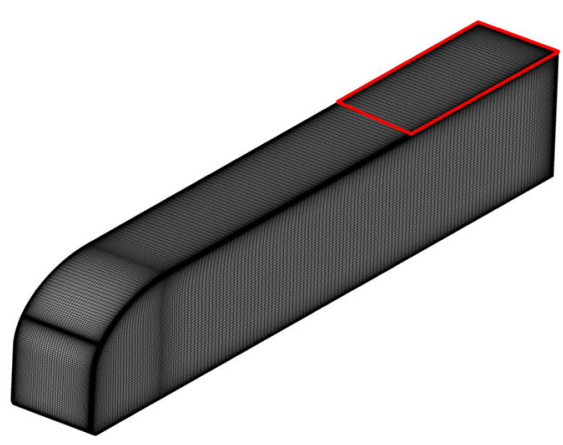

(a)

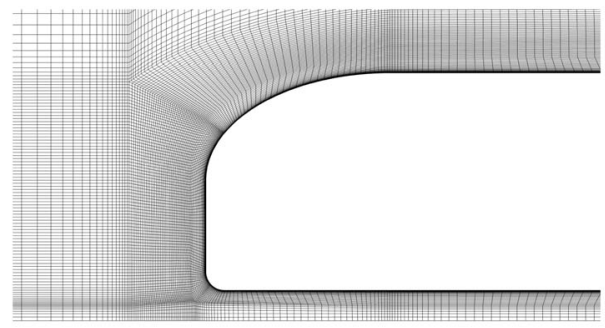

(c)

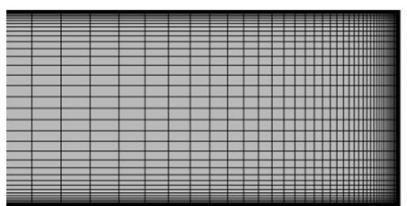

(e)

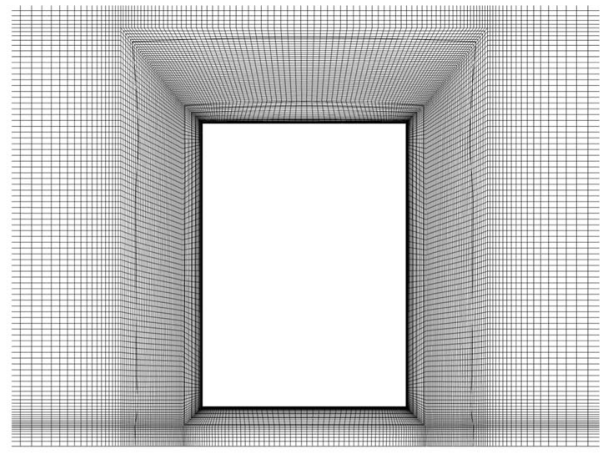

(b)

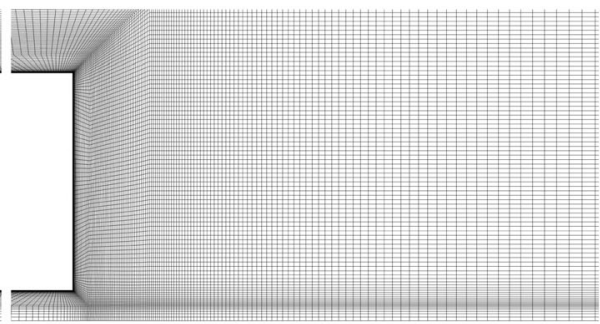

(d)

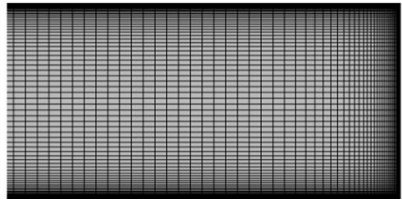

(f)

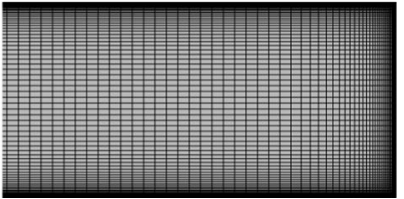

(g)

Fig. 2 a-d Visualisation of the fine mesh: a surface mesh around the GTS, with the frontal elliptical edge located at the bottom left and the base of the GTS at the top right of the figure. Cross-section of the O-grid around the GTS in the b $Y-Z$ plane at $X / H=-0.45$, c at the front of the model in the vertical midplane and $\mathbf{d}$ at the rear of the model in the vertical midplane. Close-up of the surface mesh distribution on the roof of the GTS is marked by a red outline in (a) for the e coarse mesh, $\mathbf{f}$ medium mesh and $\mathbf{g}$ fine mesh

distribution of elements on the fine mesh on the GTS, while Fig. $2 b-d$ show the crosssection of the mesh in the $Y-Z$ plane, and in the vertical midplane of the model at the front and the rear. The distribution of the cells is concentrated close to the walls of the GTS and in the near wake. Also seen in Fig. 2e to g are the close-up views of the roof of the GTS for the coarse, medium and fine meshes, respectively. While a reliable LES grid is expected to resolve $\simeq 80 \%$ of the turbulent kinetic energy everywhere in the flow field [62], the computational requirements to resolve the wall boundary layer on non-critical bodies/surfaces such as the ground plane which extends to large distances away from the body, are exceedingly high. In this regard, the boundary layer on the no-slip wall (and the slip wall) was modelled with a the first element in the wall-normal direction placed slightly above $n^{+}=30$; while $n^{+}=1$ was used to resolve the boundary layer on the no-slip walls of the GTS model. Here, $n^{+}$is the dimensionless wall distance given by $n^{+}=n u_{\tau} / \nu$, where, $n$ is the distance of the first cell height from the wall, and $u_{\tau}$ is the friction velocity. Furthermore, the recommended grid spacing in the streamwise and spanwise directions must be of order 
Table 1 Details of the computational mesh

\begin{tabular}{lclll}
\hline Description & Number of elements (million) & $n_{\text {mean }}^{+}$ & $\Delta s_{\text {max }}^{+}$ & $\Delta l_{\text {max }}^{+}$ \\
\hline Coarse & 3.88 & 0.75 & 195 & 75 \\
Medium & 8.35 & 0.5 & 46 & 25 \\
Fine & 10.78 & 0.4 & 36 & 20 \\
\hline
\end{tabular}

$\Delta s^{+}=\Delta s u_{\tau} / v=50-150, \Delta l^{+}=\Delta l u_{\tau} / v=15-40$, respectively, in order to resolve the near-wall structures (see [60]). Here, $\Delta s$ and $\Delta l$ are the grid spacings in the streamwise and spanwise directions, respectively. Table 1 shows the mean grid spacing in the wallnormal direction and the maximum grid spacing in the streamwise and lateral directions. The medium and the fine meshes are well below the suggested limits, while the coarse mesh exceeds them. Although the $n^{+}$was higher than unity around the sharp edge side corners and the bottom filleted surface at the front of the model, the values never exceeded $n^{+}=2.5$.

For all the cases considered in this study, the time-step used was set to $7.5 \times 10^{-4} s$ to ensure a CFL (Courant-Friedrichs-Lewy) number less than unity around the GTS model. All simulations were started from a quiescent condition, and the averaging of the flow quantities and forces was carried out after one flow passage for five flow passages through the domain. For the computation of the force coefficients, the summation of the viscous and pressure forces in each direction was divided by $0.5 \rho U_{\infty}^{2} A$, where $\rho$ is the density of air $\left(=1.1885 \mathrm{kgm}^{-3}\right)$, and $A$ is the frontal area of the GTS $\simeq 0.146 \mathrm{~m}^{2}$. The difference in the drag force coefficient between the fine and medium mesh was less than $2.25 \%$ and $3 \%$ for the incoming flow at yaw angles of $0^{\circ}$ and $10^{\circ}$, respectively (see Section 3.3). To further investigate the influence of the spatial resolution of the meshes used, the time-averaged flow fields were compared at locations downstream of the base. Shown in Fig. 3a and b are streamwise and vertical velocity profiles at the specified locations in the near wake. In each of these images, the $x$ coordinate for the velocity profiles is obtained by the following relation: $X=x_{o}+\left(\left(u_{x, z} / U_{\infty}\right)-1\right) \times 0.1$. Here, $x_{o}$ is the $x$ coordinate (with the origin at the base), $u_{x}$, and $u_{z}$ are the values of mean streamwise and vertical velocities, respectively. The velocity profiles were measured at intervals of $0.25 H$ from the base to $X / H=2$. At locations close to the base of the GTS, all the three meshes capture the velocity profiles accurately, and at a location $X / H \simeq 1$, the discrepancy between the medium and the fine meshes is larger, with the fine mesh closer to the coarse mesh. This discrepancy reduces at larger streamwise distances from the base. Furthermore, the locations of the centre of the vortices were compared (see Fig. 6), which further show that the location of the vortex centres of the medium mesh are closer to the base as compared to the fine mesh.

\section{Results}

\section{$3.1 \beta=0^{\circ}$}

In this section, flow past a simplified GTS model is investigated at $R e_{W} \simeq 2.7 \times 10^{4}$ for $\beta=0^{\circ}$. Shown in Fig. $4 \mathrm{a}$ and $\mathrm{d}$ are the contours of the time-averaged and instantaneous flow at the front of the model in the vertical midplane, respectively. The stagnation point occurs at height of $\simeq 0.31 \mathrm{H}$ from the bottom edge of the model and the flow remains attached over the elliptical shaped nose, and beyond this region, a very small recirculation region 


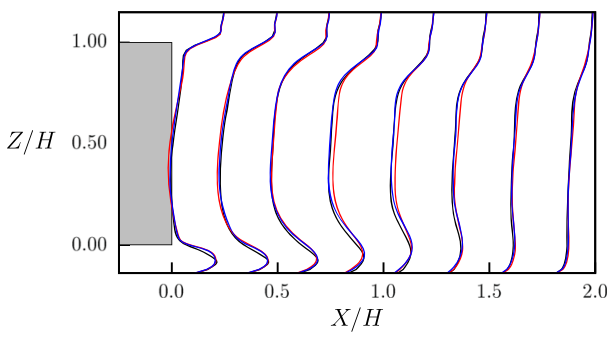

(a)

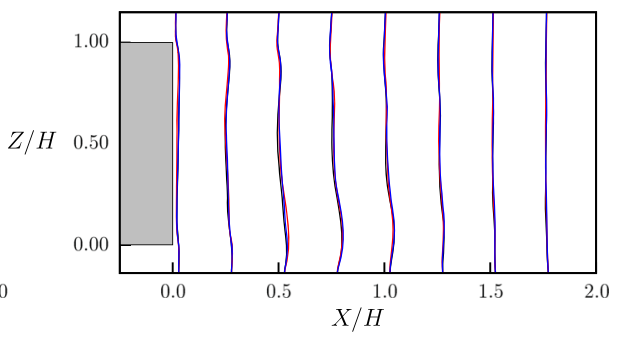

(b)

Fig. 3 Comparison of the velocity profiles in the wake of the GTS model in the vertical midplane. a streamwise velocity and $\mathbf{b}$ vertical velocity. The black, red and blue lines correspond to the coarse, medium and fine mesh, respectively. Flow is from left to right in these images

is observed, beyond which undulations in the flow are observed. This region is outside of the PIV window used in the experimental work of [45], where the flow remained attached over the nose of the model. On the sides of the model, a fixed separation point is observed on account of the sharp frontal edge (Fig. 4b), resulting in a recirculation zone that extends $\simeq 0.95 \mathrm{H}$ from the front, and is nearly twice longer as that reported in [45]. Also observed in Fig. $4 \mathrm{~b}$ is a smaller recirculating adjacent to the wall. Although the size of this structure is rather small for the incoming flow at zero yaw angle, this recirculating region is larger as the yaw angle increases (also see Fig. 10d - on the right side of the GTS - or on the upper side in plan view). In the instantaneous frame (Fig. 4e), a train of discrete vortices is observed that dissipate further downstream. A train of vortices is also observed on either side of the longitudinal symmetry plane in the wake as seen in Fig. $4 \mathrm{f}$, at the rear of the GTS. However, in the mean flow, two counter-rotating vortices are observed in the lateral midplane (Fig. 4c). The location of the centre of these vortices and the saddle point are $\simeq 0.2 \mathrm{H}$ further downstream as compared to their experimental counterparts.

Of interest here is the near-wake topology in the vertical midplane where the flow topology of the time-averaged flow is anti-symmetric when compared to the studies of [45] and [71]; but is similar to that observed in the wake of a Generic European Transport System (GETS) model, which has a height-to-width ratio similar to the GTS - 1.35 (see Fig. 11.8 of [74]). Shown in Fig. 5a and b are the time-averaged flow topology in the near wake of the GTS model at a gap height of $0.14 \mathrm{H}$ and $1.1 \mathrm{H}$, respectively, while their counterparts of the instantaneous flow fields are shown in Fig. 5c and d, respectively. While the larger triangular-shaped vortex appears closer to the ground plane in the experiments, it appears above the lateral midplane in these numerical simulations, with the smaller elliptical-shaped vortex appearing closer to the ground plane. It may be recalled that bi-stability was observed in the lateral plane of a squareback Ahmed body, where the width-to-height ratio is approximately equal to the height-to width ratio of the GTS model ([5, 20-22, 33, 38, 39, 58, 59, 76, 78 ] and others). Given the close match of the centres of the vortex locations in Fig. 6a, the other bi-stable flow state is observed in these simulations. Recent experimental observations in the near wake of a simplified truck model (whose height-to-width ratio is 1.35) confirmed the existence of this flow state (see [67]). Furthermore, the flow topology is invariant to the change in the gap height as seen in Fig. 5b, as a near identical flow topology is observed in the vertical midplane at a gap height of $G / H=1.1$. This is further confirmed by the close match of the vortex centre locations at gap heights of $0.14 \mathrm{H}$ and $1.1 \mathrm{H}$ as shown in Fig. 6 $[20,45]$. Thus, the occurrence of bi-stable states is not dependent on the gap height, but on the $H / W$ ratio of the model [21] for $G / H \geqslant 0.14$. The anti-symmetric flow field for the 


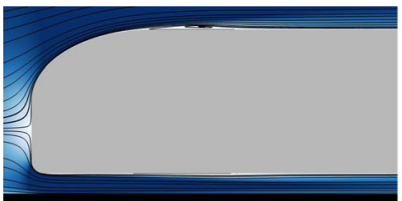

(a)

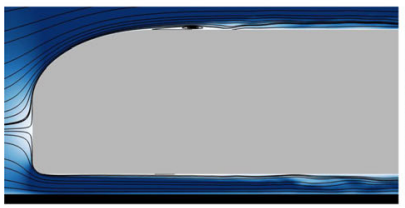

(d)

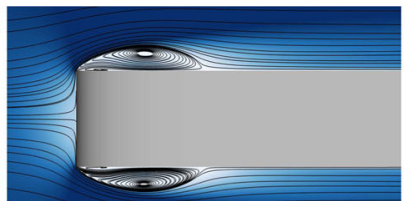

(b)

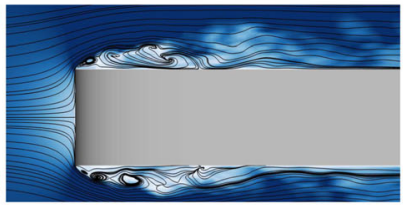

(e)

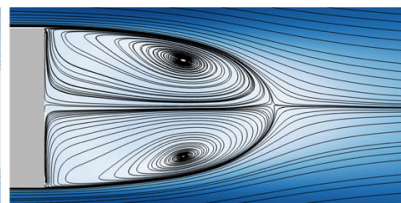

(c)

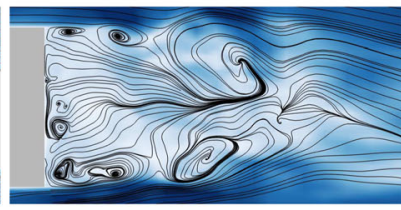

(f)

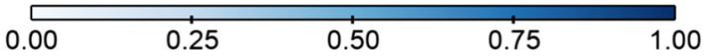

Fig. 4 Visualisation of the contours of the normalised time-averaged velocity $(\mathbf{a}-\mathbf{c})$ and normalised instantaneous velocity $(\mathbf{d}-\mathbf{f})$ overlaid with streamlines at the front of the GTS model in the vertical midplane - a, $\mathbf{d}$, the lateral midplane - $\mathbf{b}, \mathbf{e}$, and at the rear of the model in the lateral midplane - c, f. Flow is from left to right in these images

GTS model at $G / H=0.14$ leads to flow separation on the ground plane directly below the saddle point of the flow, similar to that observed in [49]. Furthermore, a large amount of downwash is generated in this flow state, and perhaps suppresses any increase in the height of the ground vortex (Fig. 5a).

Figure $6 \mathrm{a}$ and $\mathrm{b}$ show the location of the centre of the vortices in the vertical and lateral midplanes, respectively. For comparison, the vortex locations from the experimental works of [71] and [45] are also shown. Despite the large difference in the Reynolds number, the locations of the vortex centres in the vertical midplane are in good agreement with their experimental counterparts. Vortex A corresponds to the tiny bottom vortex adjoining the large triangular-shaped vortex (B), while the smaller (and elliptical-shaped) vortex (C) is observed on the opposite side of the large triangular vortex. In the lateral midplane, two symmetrical counter-rotating vortices are observed $\left(\mathrm{D}_{R}\right.$ and $\mathrm{D}_{L}$, corresponding to the right and left vortices). The saddle points in the vertical and lateral midplanes are denoted by $S_{1}$ and $S_{2}$, respectively. Also shown in this figure are the centres of the vortices for the GTS model at a height of $1.1 \mathrm{H}$ from the ground. The results show that the locations of the vortex centres of the medium mesh are closer to the base as compared to the coarse and fine mesh, while those observed for the gap height of $1.1 \mathrm{H}$ are closer to its counterparts of the fine mesh for the GTS at a gap height of $0.14 \mathrm{H}$. In the lateral midplane, the vortex centres are located further downstream as compared to the experimental work of [45].

The analysis of the near wake is considered in Fig. 7 by the visualising the vortex contours overlaid with streamlines computed from the mean flow in the specified planes. Figure 7a shows the formation of the stagnation point at a height of $0.25 \mathrm{H}$ from the bottom of the base in the $Y-Z$ plane, which is much higher than that reported in [45]. Also discernible are the two counter-rotating vortices close to the ground (bottom of the image), which originate at the front of the model. At downstream distances of $0.225 \lesssim X / H \lesssim 0.6$ from the base, a counter-rotating vortex pair is observed; whose vortex centres occur closer to the bottom edge of the base as the downstream distance is increased. This corresponds to the ring vortex that has been observed in previous studies (also see Fig. 14a), and is observed for $X / H \leqslant 1$, which is within the confines of the recirculation zone in the near wake. 


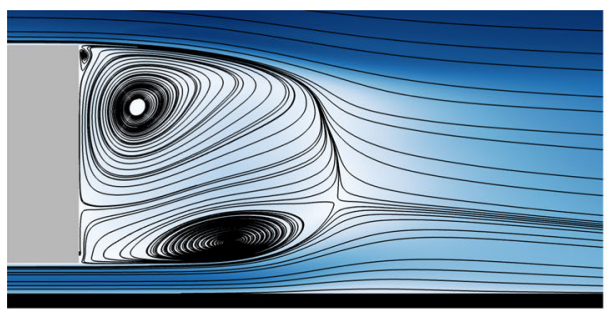

(a)

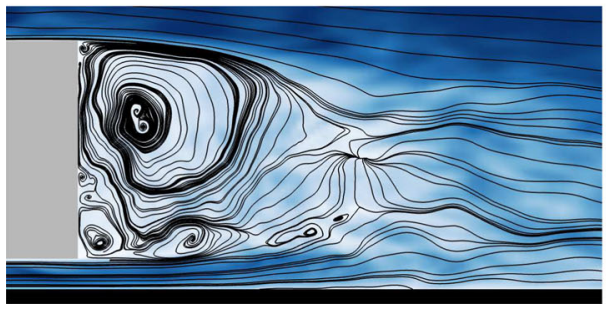

(c)

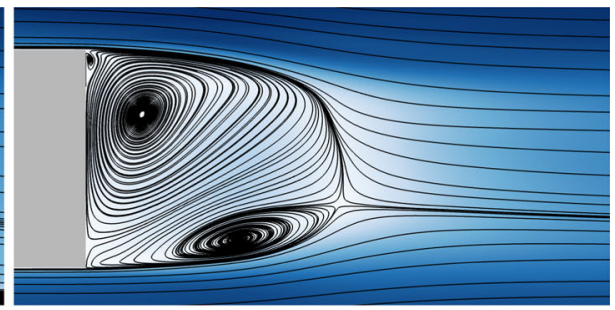

(b)

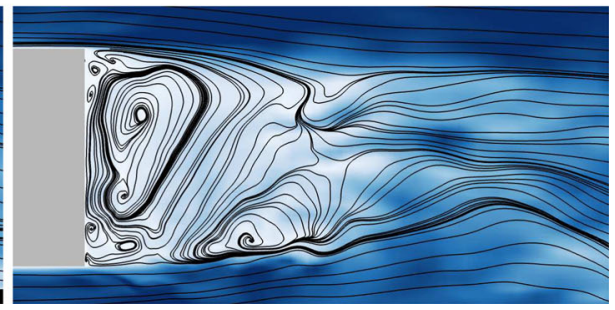

(d)

Fig. 5 Visualisation of the contours of the normalised time-averaged velocity - $\mathbf{a}, \mathbf{b}$ and normalised instantaneous velocity - c, $\mathbf{d}$ overlaid with streamlines at the rear of the GTS model in the vertical midplane for a gap height a, $\mathbf{c} G / H=0.14$, and $\mathbf{b}, \mathbf{d} G / H=1.1$. Flow is from left to right in these images. Contour shading as per Fig. 4

Note that Fig. 7b is similar to Fig. 16 of [23], albeit transposed across the lateral midplane, where an anti-symmetric flow topology is observed in the vertical midplane in their URANS simulations as compared to the current study. In the $X-Y$ planes (Fig. $7 \mathrm{~g}-1$ ), the counterrotating vortex pair is observed for $0.15 \lesssim Z / H \lesssim 0.7$, as the planes slice across the ring vortex. The centres of the two counter-rotating vortex pairs are observed at distances farther away from the base at locations close to the bottom of the base, and gradually move closer to the base at higher locations. The length of the recirculation zone decreases marginally from $Z / H=0.33$ to $Z / H=0.69$. At $Z / H=0.69$, a saddle point close to the base is observed at a streamwise distance of $X / H \simeq 0.15$. Beyond $Z / H=0.75$, the streamlines are in-line with the incoming flow. A pair of vortices along the base and close to the outer edges (parallel to the longer edges of the model) are observed in the $X-Y$ planes, and these become prominent at $Z / H \simeq 0.83$ (also see Figs. 9 and 10 of [33]). It may be noted that vortical structures shown here are not perfectly symmetrical across the $Y=0$ symmetry plane, possibly due to the relatively shorter averaging periods as compared to previous experimental studies.

The contours of the Reynolds normal stresses in the vertical and lateral midplanes are shown in Fig. 8 with the contour levels set to those used in [45]. In the vertical midplane, the maximum value of the normal stresses in the streamwise direction $\left(\left\langle u_{x}^{\prime 2}\right\rangle\right)$ occurs close to the smaller elliptical-shaped vortex located close to the ground, and also at locations diametrically opposite to it, but at a slightly lower intensity; and stretching slightly downstream of the recirculation zone (Fig. 8a). In the lateral midplane, it occurs along the outer edges of the counter-rotating vortices as seen in Fig. 8d. The Reynolds normal stresses in the spanwise direction occur above the saddle point and in the region that divides the inner and outer regions of the time-averaged flow in the vertical midplane as seen in Fig. 8b, while those in the vertical direction occur around the saddle point region (Fig. 8c). In the lateral midplane, 


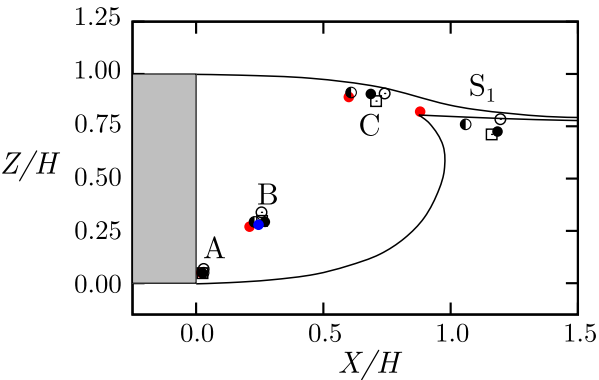

(a)

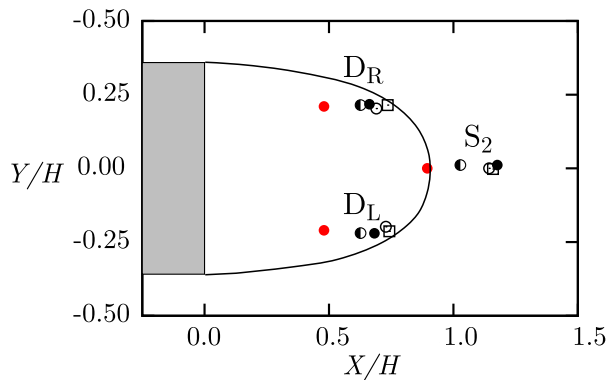

(b)

Fig. 6 Location of the centre of the vortices obtained from the time-averaged wake of the GTS in a the vertical midplane and $\mathbf{b}$ lateral midplane. While the flow topology in the LES simulations were anti-symmetrical to those observed in the experiments, the vortex locations were inverted to compare directly with previous experimental studies. For each vortex location and the saddle point, the coarse mesh $(\bigcirc)$, medium mesh

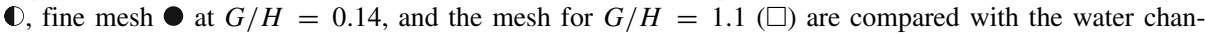
nel experiments of [45] at an identical Reynolds number and the wind tunnel experiments of [71] at $R e_{W}=2 \times 10^{6}$. The bounding streamlines (separatrix) from [45] are also marked by black continuous lines. Flow is from left to right in these images

the distribution of the normal stresses in the spanwise and vertical direction occurs in the region of the saddle point as seen in Fig. 8e and f. These images compare well with Fig. 9 of [45], and Figs. 6 and 7 of [21].

Shown in Fig. 9a - d are the power spectral densities (PSD) of the streamwise, spanwise, vertical velocities and the pressure at a point in the wake $(X / H, Y / H, Z / H)=$ $(1.67,0,0.5)$, which is downstream of the recirculating region. The streamwise component

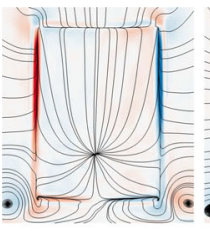

(a)

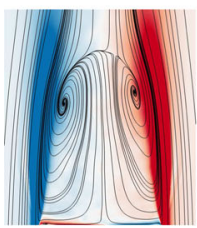

(g)

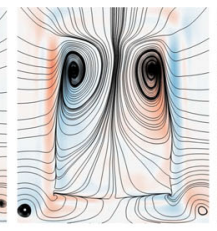

(b)

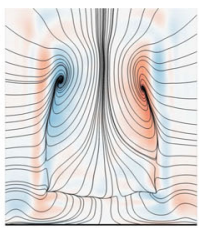

(c)

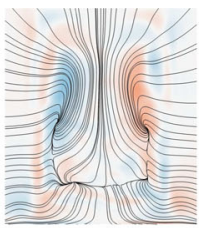

(d)

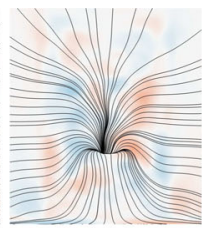

(e)

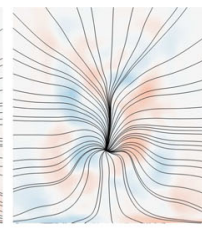

(f)

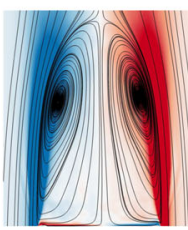

(h)

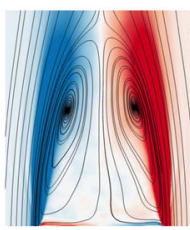

(i)

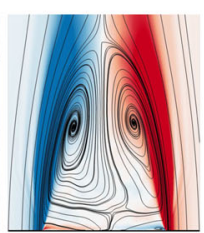

(j)

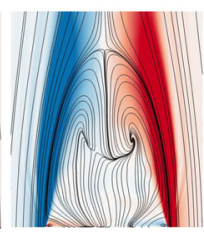

(k)

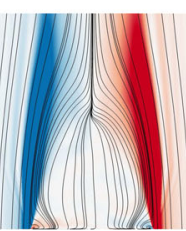

(1)

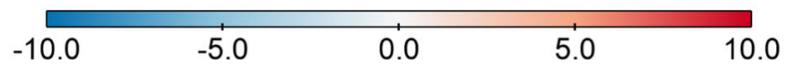

Fig. 7 Visualisation of the contours of the time-averaged streamwise vorticity $\left(\omega_{x}\right)(\mathbf{a})-(\mathbf{f})$, and vertical vorticity $\left(\omega_{z}\right)(\mathbf{g})-(\mathbf{l})$ overlaid with streamlines at the specified locations for the GTS model for $\beta=0^{\circ}: \mathbf{a}$ $X / H=0.1, \mathbf{b} X / H=0.333$, b $X / H=0.5$, d $X / H=0.66$, e $X / H=1, \mathbf{f} X / H=1.25, \mathbf{g} Z / H=0.17, \mathbf{h}$ $Z / H=0.33, \mathbf{i} Z / H=0.6, \mathbf{j} Z / H=0.69, \mathbf{k} Z / H=0.75$ and $\mathbf{l} Z / H=0.83$. Figures (a) $-(\mathbf{f})$ are viewed from behind the GTS model, while figures $\mathbf{g}-\mathbf{l}$ are in plan view with flow direction from bottom to top in each image 


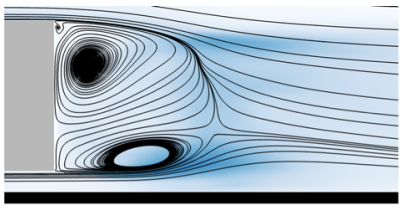

(a)

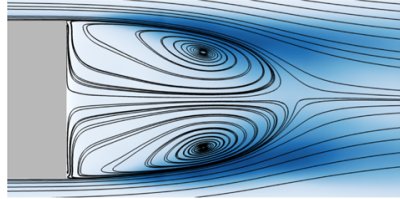

(d)

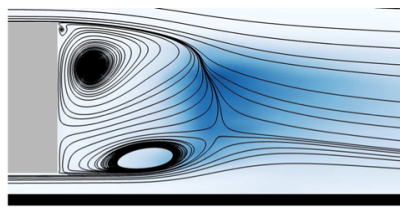

(b)

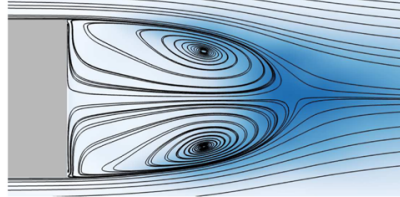

(e)

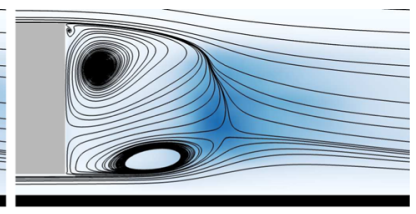

(c)

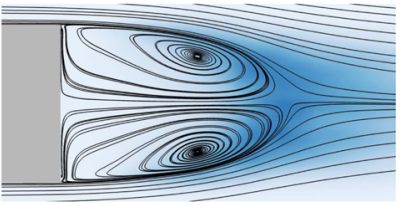

(f)

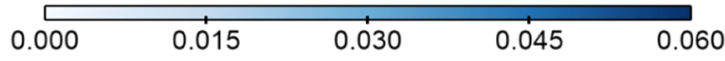

Fig. 8 Visualisation of the contours of the Reynolds normal stresses at the rear of the GTS model overlaid by streamlines in the vertical midplane $(\mathbf{a}-\mathbf{c})$, and in the lateral midplane $(\mathbf{d}-\mathbf{f}) .\left\langle u_{x}^{\prime 2}\right\rangle-(\mathbf{a})$ and $(\mathbf{d}),\left\langle u_{y}^{\prime 2}\right\rangle-$ (b) and (e), and $\left\langle u_{z}^{\prime 2}\right\rangle-(\mathbf{c})$ and (f). Flow is from left to right in these images

clearly shows the wake pumping frequency of $S t_{W}=0.08$ reported in previous studies [3, $13,33,45,78]$, which corresponds to the lengthening and shortening of the wake. The spanwise and streamwise components show a peak around $S t_{W}=0.2$, which is consistent with the observations of [78] (see Fig. 16c of their article) and [75]. The PSD of the pressure shows a peak around $S t_{W}=0.17$ (Fig. 9d), which is consistent with the observations of [27] and [21] when sampled at a point in the symmetry plane beyond the recirculating region.

\subsection{Influence of the yaw angle $\left(\beta>0^{\circ}\right)$}

In this section, we investigate the changes brought about by an incoming flow at yaw or a constant crosswind. An understanding of the flow topology and the forces obtained here could be used to minimise any adverse effects that may be brought about by crosswinds such as stability of the vehicle (also see [79]). [76] reported the percentage of time the two bi-stables states were observed for a squareback Ahmed body. When the body was at a yaw angle of $0^{\circ}$, the ratio of the occurrence of the dominant larger triangular-shaped vortex on the left-to-right side was $28: 72$, and when yawed by $0.35^{\circ}$, the ratio was $90: 10$, indicating the sensitivity to the yaw angle. Here, the incoming flow was yawed by $\beta=2.5^{\circ}$ to investigate if the slight asymmetry of the incoming flow could trigger the other bi-stable flow state. Shown in Fig. 10 are the contours of the flow field in the vertical and lateral midplanes at the front and the rear of the GTS model for $\beta=2.5^{\circ}$, and for a larger value of yaw at $\beta=10^{\circ}$. A flow topology similar to the non-yawed case was observed in the vertical midplane in the near wake for $\beta=2.5^{\circ}$, indicating that the flow is rather insensitive to small values of yaw angles. At a larger yaw angle of $\beta=10^{\circ}$, the flow topology is nearly symmetrical with the rear stagnation point midway between the base, and the saddle point above the lateral midplane (Fig. 10e). In the lateral midplane, a larger recirculation region develops on the right side at the front of the GTS, which grows larger as the yaw angle is increased as seen in Fig. 10d for $\beta=10^{\circ}$. Comparing this image to Fig. 5 of [43], the differences brought about by the sharp frontal edge can be observed. While a smaller recirculation region persists on the front left side of the image in the current work; the flow remains attached for the 


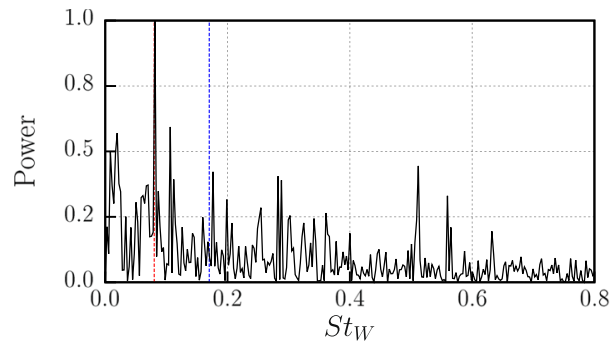

(a)

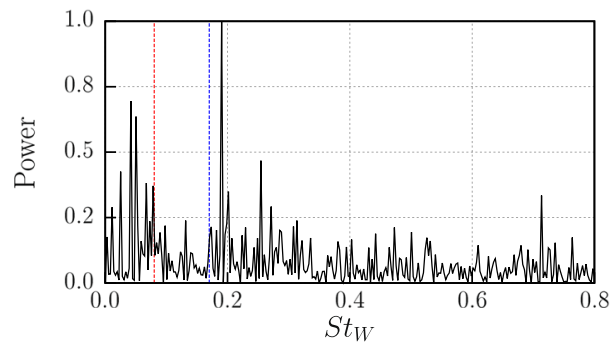

(c)

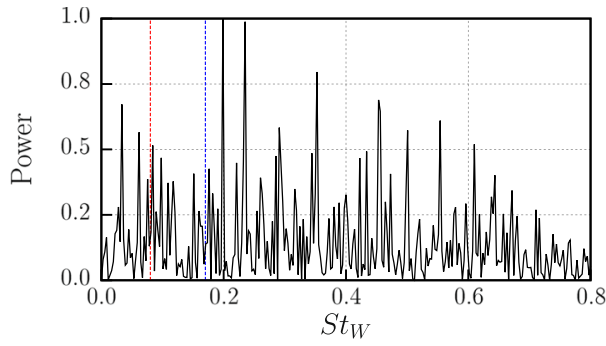

(b)

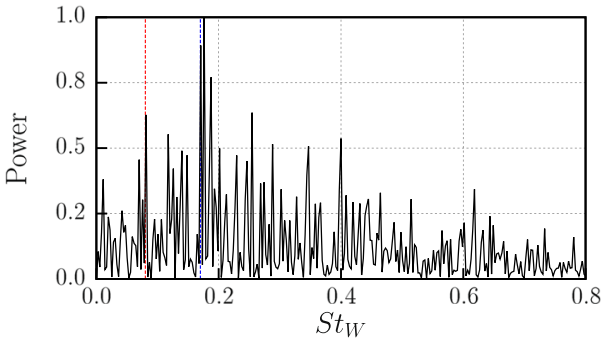

(d)

Fig. 9 PSD of a point in the wake at $(X / H, Y / H, Z / H)=(1.67,0,0.5)$ for a streamwise velocity, b spanwise velocity, c vertical velocity and $\mathbf{d}$ pressure. The red and blue lines correspond to Strouhal numbers of 0.08 and 0.17 , respectively

filleted A-pillar. At the rear of the model, yawing brings about asymmetry, with the saddle point closer to the direction of yawing. As the yaw angle is increased from $\beta=2.5^{\circ}$ to $\beta=10^{\circ}$, the flow topology in the lateral plane does not change significantly, although the rear stagnation point (closer to the base) moves closer towards the edge of the model in the direction of incoming flow. Nonetheless, these images compare well (qualitatively) with a

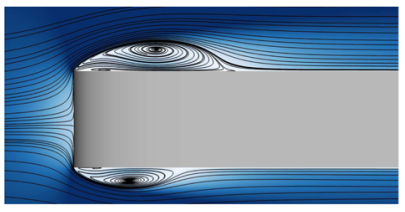

(a)

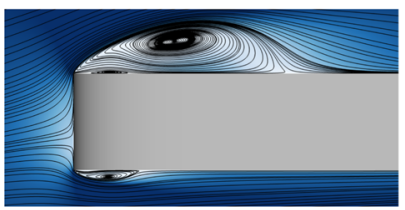

(d)

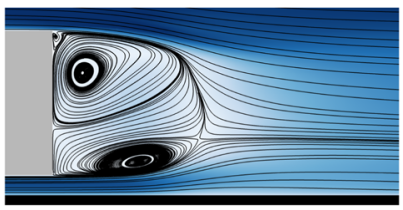

(b)

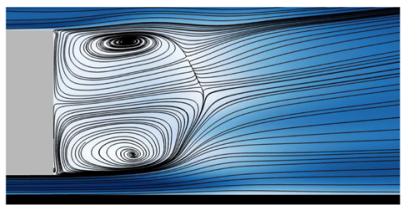

(e)

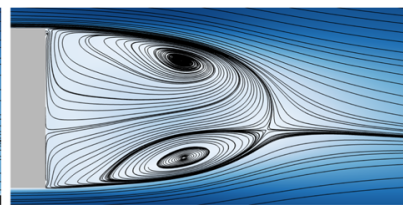

(c)

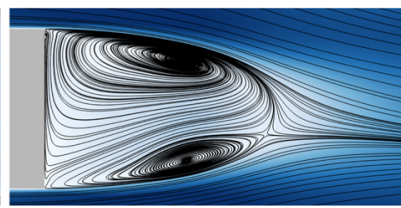

(f)

Fig. 10 Visualisation of the contours of the normalised time-averaged velocity overlaid with streamlines for $\beta=2.5^{\circ}-(\mathbf{a})-(\mathbf{c})$, and $\beta=10^{\circ}$ (d) - (f). Flow in the lateral midplane at the front of the GTS model is shown in images (a) and (d), and in the vertical midplane at the rear is shown in images (b) and (e). The flow in the lateral midplane at the rear of the model is shown in images (c) and (f). Flow is from left to right in all these images. Contour shading as per Fig. 4 


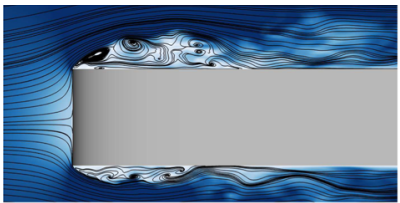

(a)

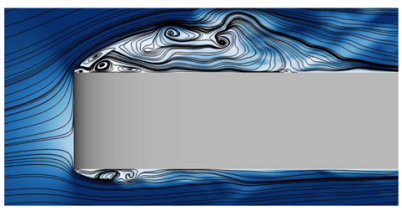

(d)

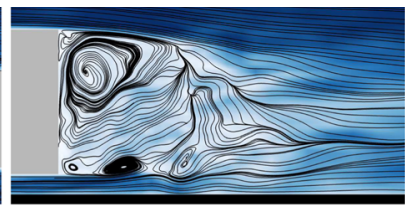

(b)

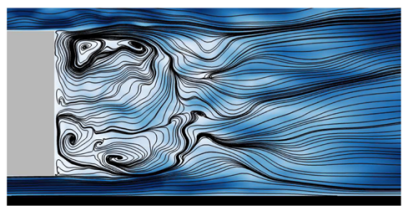

(e)

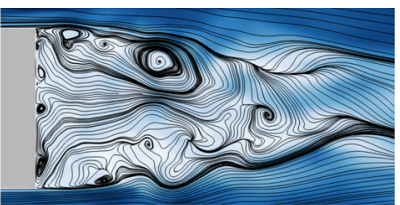

(c)

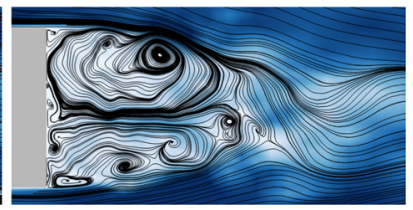

(f)

Fig. 11 Visualisation of the contours of the normalised instantaneous velocity overlaid with streamlines for $\beta=2.5^{\circ}-(\mathbf{a})-(\mathbf{c})$, and $\beta=10^{\circ}$ (d) - (f). Flow in the lateral midplane at the front of the GTS model is shown in images (a) and (d), and in the vertical midplane at the rear is shown in images (b) and (e). The flow in the lateral midplane at the rear of the model is shown in images (c) and (f). Flow is from left to right in all these images. Contour shading as per Fig. 4

previous prediction using RANS [65, 70] and LES for a real truck ([11]; also see Figs. 11 and 12 of [74]).

Figure 11 shows the instantaneous velocity contours overlaid with streamlines at the specified planes for the two yaw angles considered in Fig. 10. The location of the vortices/flow structures observed in these figures is consistent with those observed in the mean flow topologies (Fig. 10). At the rear of the GTS, the side of the larger of the two vortices observed in the mean flow topology in the lateral and vertical midplanes consists of a single large vortex in the instantaneous snapshots; while a series of smaller vortices are observed on the side where the smaller vortex occurs in the mean flow. The side of the smaller vortex is also associated with the region of higher Reynolds stresses in the streamwise direction (see Fig. 15).

Figure 12 shows the surface flow visualisations on the right hand side of the GTS for the specified yaw angles. The nodal points $(\mathrm{N})$ and saddle points $(\mathrm{S})$ have been identified for each case (also see [25, 41]). For $\beta=0^{\circ}$, an initial separation line is observed close to the frontal edge of the model, which marks the onset of the recirculation region adjacent to the wall and ends with the line joining nodal points N1 and N2 with saddle point S1; while the larger recirculation zone extends approximately to a distance equal to the height of the GTS at $Z / H=0.5$, as seen in Fig. 4b. This larger recirculation zone forms a near parabolic separation line, with the vertex of the parabola situated close to the lateral midplane of the body. A second saddle point - S2 is observed slightly below the midplane of the GTS. Beyond this saddle point, the flow traverses along the side of the GTS, and away from the nodal points $\mathrm{N} 3$ and $\mathrm{N} 4$. For $\beta=2.5^{\circ}$, the surface flow is similar to the $\beta=0^{\circ}$ case, with the lengths of the separation zones having increased in the streamwise direction, and no saddle points observed. At distances closer to the base of the model, the separation lines formed by the corner vortices close to the bottom and the top edges are visible, and they grow larger as the yaw angle is increased to $\beta=10^{\circ}$. The frontal separation extends to approximately $1.5 \mathrm{H}$ downstream of the frontal edge for $\beta=10^{\circ}$, while a distinct separation bubble is not observed in the RANS simulation of [65] at front of the GTS model on account of the rounded/curved A-pillars of the GTS model.

Figure 13 shows the contours of the pressure coefficient $C_{p}=2\left(p-p_{\infty}\right) / \rho U_{\infty}^{2}$, on the base of the GTS model at the specified yaw angles. For $\beta=0^{\circ}$, a region of low pressure 
is observed in the upper part of the base, where the larger triangular vortex is present. The close proximity of this larger vortex is responsible for the low-pressure region (also see Fig. 14a, where the vortex core is closer to the base above the lateral midplane), while a region of slightly higher pressure is observed at the bottom of the base. It may be recalled that the centre of the bottom vortex occurs further away from the base. This can further be confirmed by the visualisation of the isosurfaces of the pressure coefficient as seen in Fig. 14a. In the other bi-stable state, the low and high pressure regions occur at bottom and top of the base, respectively. Furthermore, the contours are very similar to those observed behind a squareback Ahmed body, although rotated by $90^{\circ}$ (also see Fig. 7a of [42]). As the yaw angle is increased to $\beta=2.5^{\circ}$, the region of lower pressure at the top of the base increases, along with the formation of a low pressure region on the right side (also see Fig. 11.11 of [74]). On further increasing the yaw angle to $\beta=10^{\circ}$, a larger region of low pressure is observed at the top and the distribution of the pressure is nearly symmetrical about the lateral midplane, with the low-pressure regions occurring on the right side of the base, away from the direction of the incoming flow.

Figure 14 shows the translucent isosurfaces of the pressure coefficient $\left(C_{p}\right)$ behind the base of the GTS model at the specified yaw angles of the incoming flow, overlaid with the vortex cores obtained from the time-averaged flow by using the method detailed in [72]. Similar to the pressure coefficient torus observed in the wake of squareback bluff bodies ([2, $15,21,33,42,77]$ and others), a torus tilted in the streamwise direction is observed behind the GTS for $\beta=0^{\circ}$ (Fig. 14a), with the bottom of the torus further away from the top on account of the flow asymmetry (and where the smaller vortex (C) occurs in the vertical midplane). This structure is similar to that observed in the wake of a squareback Ahmed body (see Fig. 3a of [42]) - where the flow asymmetry is in the lateral midplane, with the small vortex occurring at the location where the torus is farthest from the base. It maybe

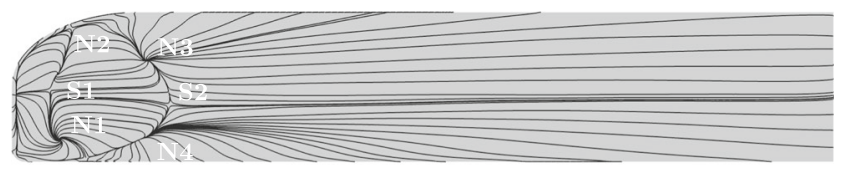

(a)

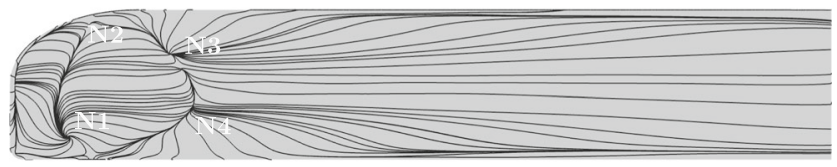

(b)

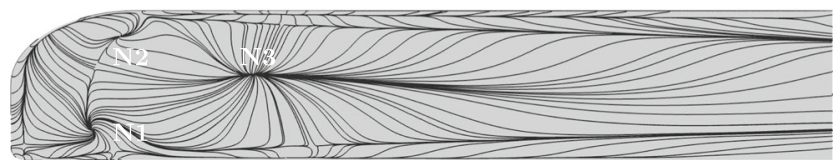

(c)

Fig. 12 Visualisation of the time-averaged surface streamlines on the right side of the GTS, away from the incoming flow for the yaw angles of $\mathbf{a} \beta=0^{\circ}, \mathbf{b} \beta=2.5^{\circ}$ and $\mathbf{c} \beta=10^{\circ}$. The nodal points are marked by a capital letter $\mathrm{N}$, while the saddle points are marked by a capital letter S. Flow is from left to right in these images 


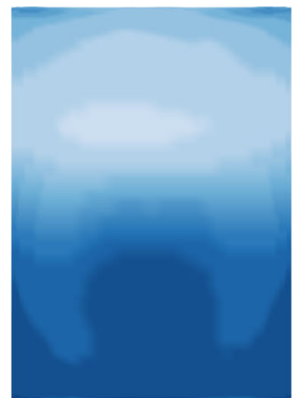

(a)

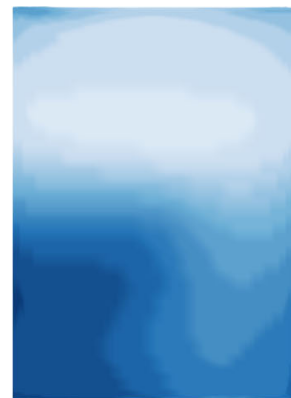

(b)

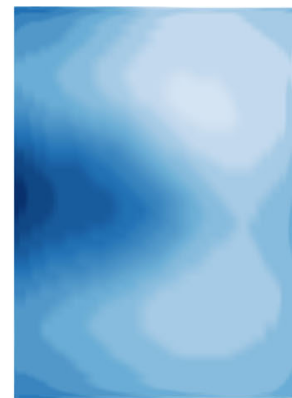

(c)

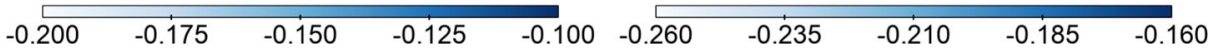

Fig. 13 Visualisation of the contours of pressure coefficient on the base of the GTS model for a $\beta=0^{\circ}$, b $\beta=2.5^{\circ}$, and c $\beta=10^{\circ}$. Contour levels for each image are chosen to accentuate the variation in the pressure coefficient. Contour levels in (a) and (b) are between $-0.2 \leqslant C_{p} \leqslant-0.1$, and between $-0.26 \leqslant$ $C_{p} \leqslant-0.16$ in $(\mathbf{c})$

recalled that the averaging of the pressure quantities are performed over one flow state, and thus results in the formation of a torus titled in the streamwise direction. This is in contrast to the ring vortex (or torus) in the wake of a squareback Ahmed body being in a plane parallel to the base, as seen in Fig. 5 of [21], when averaged over the two stable flow states. This asymmetry is also demonstrated by the isosurfaces of the velocity when conditionally averaged over one flow state, while the distribution is symmetric when averaged over the two flow states [76, 78].

Figure $14 \mathrm{~b}$ and $\mathrm{c}$ show the changes to the shape of the pressure coefficient torus brought about by the incoming flow at yaw angles of $\beta=2.5^{\circ}$ and $10^{\circ}$, respectively. While the shape of the torus at $\beta=2.5^{\circ}$ is similar to $\beta=0^{\circ}$, the left side of the torus (when view from a point directly behind the base) is slightly larger as compared to the right side on account of the flow asymmetry. However, for $\beta=10^{\circ}$, the flow asymmetry (in the lateral direction) is larger, with the pressure coefficient torus no longer tilted in the streamwise direction; and this is further supported by the (near) symmetrical flow topology in the vertical midplane

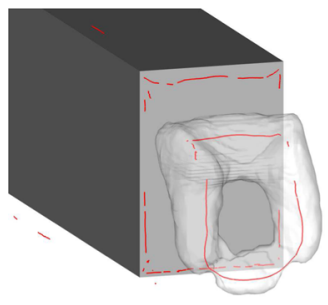

(a)

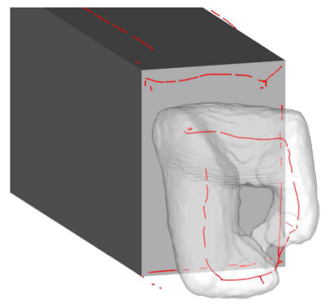

(b)

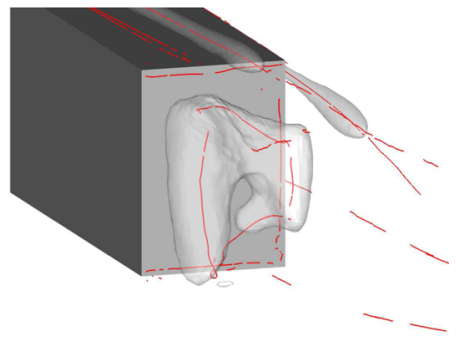

(c)

Fig. 14 Visualisation of the translucent isosurfaces of the pressure coefficient behind the GTS model for a $\beta=0^{\circ}, C_{p}=-0.2, \mathbf{b} \beta=2.5^{\circ}, C_{p}=-0.2025$, and $\mathbf{c} \beta=10^{\circ}, C_{p}=-0.2764$. The red lines indicate the location of the vortex cores obtained from the time-averaged flow field. Flow is from top left to bottom right in these images 
as seen in Fig. 10e. The vortex cores are parallel to the longer edges of the base as seen in Fig. 14c, and those along the shorter edges of the model are crescent-shaped in plan view, forming an arc between the vortex cores parallel to the longer edges. The closest point of the crescent-shaped vortex cores to the base of the model occurs close to the low pressure regions in Fig. 13c, and are nearly symmetric across the lateral midplane. Also visible in this figure are the isosurfaces of the pressure coefficient of the rooftop vortex and the right corner vortex.

The distribution of the Reynolds normal stresses for the three yaw angle cases considered in this study are shown in Fig. 15. Figure 15a - c shows the isosurfaces of the Reynolds normal stresses $\left(\left\langle u_{x, y, z}^{\prime 2}\right\rangle\right)$ in the three directions for $\beta=0^{\circ}$. Due to the anti-symmetric nature of the flow in the vertical midplane, the distribution of the Reynolds stresses in the

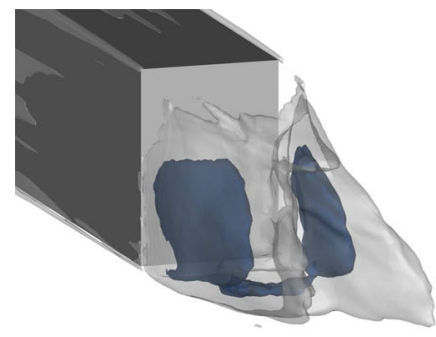

(a)

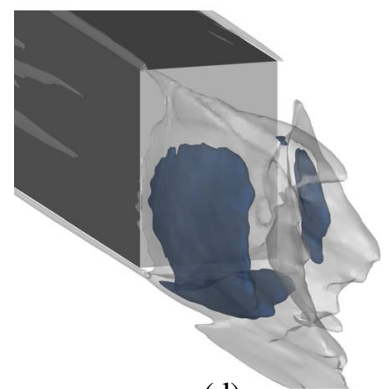

(d)

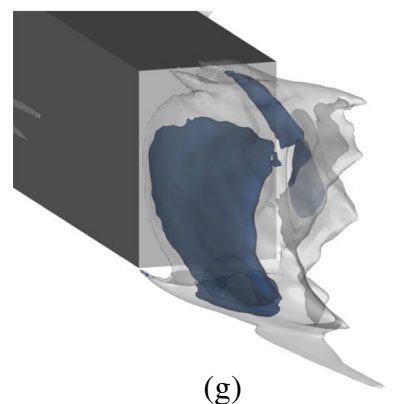

(g)

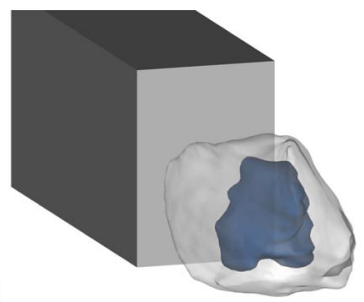

(b)

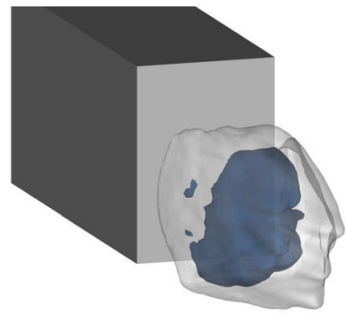

(e)

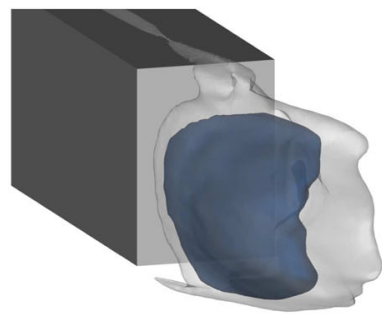

(h)

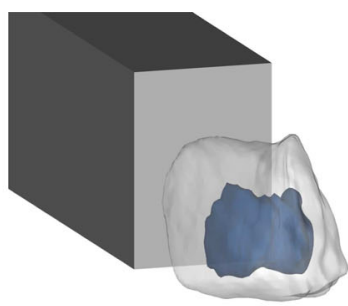

(c)

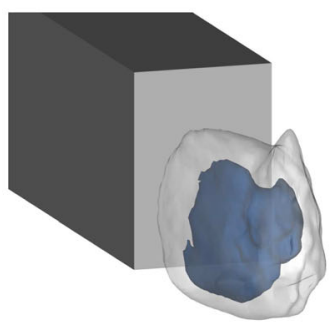

(f)

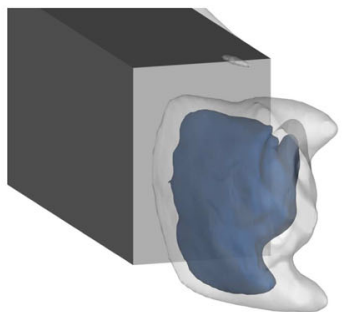

(i)

Fig. 15 Distribution of the Reynolds normal stresses behind the GTS model for a - c $\beta=0^{\circ}, \mathbf{d}-\mathbf{f} \beta=2.5^{\circ}$ and $\mathbf{g}-\mathbf{i} \beta=10^{\circ}$ for $\left\langle u_{x}^{\prime 2}\right\rangle-(\mathbf{a}),(\mathbf{d}),(\mathbf{g})\left\langle u_{y}^{\prime 2}\right\rangle-(\mathbf{b}),(\mathbf{e}),(\mathbf{h})$ and $\left\langle u_{z}^{\prime 2}\right\rangle-(\mathbf{c}),(\mathbf{f})$, (i). Isosurface levels: blue: 0.02 , white: 0.0375 . Flow is from top left to bottom right in these images 
streamwise direction are transposed across the lateral midplane as compared that reported in [45], with the maximum values occurring in the lower shear layer and along the sides, closer to the bottom edge of the base; while the distribution of stresses in the upper regions is lower. The distribution of the stresses in the other two directions occurs in a region enclosed by the distribution of the streamwise component of the Reynolds stresses. Consistent with the distribution in Fig. 8, the distribution of the normal stresses in the spanwise and vertical directions occur close to the saddle point of the flow, with the distribution of $\left\langle u_{y}^{\prime 2}\right\rangle$ extending slightly higher in the vertical direction as compared to the Reynolds normal stresses in the vertical direction $\left(\left\langle u_{z}^{\prime 2}\right\rangle\right)$. Nonetheless, the distribution of the normal stresses in the three directions is nearly symmetric across the vertical midplane.

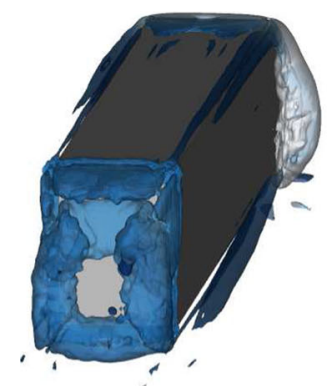

(a)

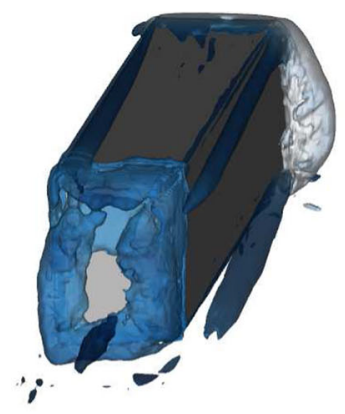

(b)

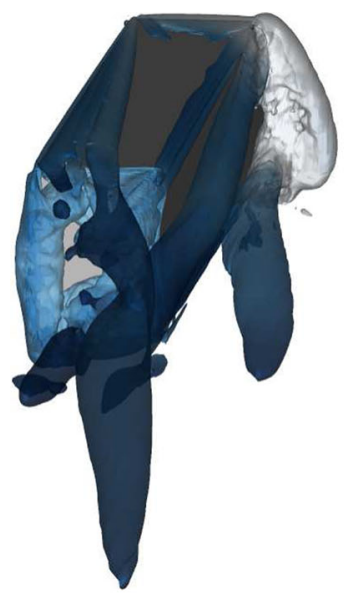

(c)

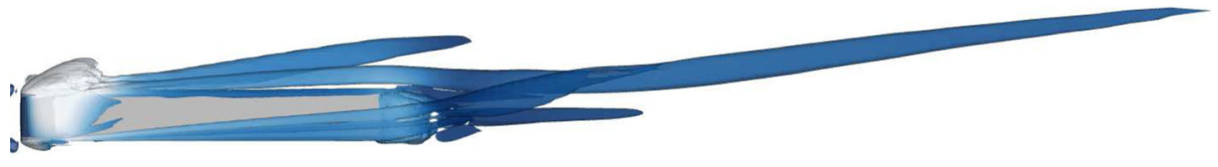

(d)

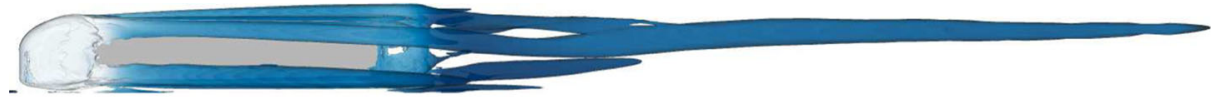

(e)

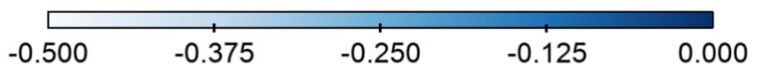

Fig. 16 Visualisation of the translucent isosurfaces of the $\lambda_{2}\left(\lambda_{2}=-2.25 s^{-2}\right)$ coloured by the contours of the pressure coefficient $\left(C_{p}\right)$ for $\mathbf{a} \beta=0^{\circ}, \mathbf{b} \beta=2.5^{\circ}$, and $\mathbf{c} \beta=10^{\circ} \mathbf{d}$ and e show image $\mathbf{c}$ in plan and (right) side views, respectively. Flow is from the top to bottom in the images (a) - (c), while it is from left to right in images (d) and (e) 
Figures $15 \mathrm{~d}-\mathrm{f}$ show the distribution of the Reynolds normal stresses in the three directions when the incoming flow is yawed at $\beta=2.5^{\circ}$. The distribution of the stresses is largely similar to that observed to the case at $\beta=0^{\circ}$, with larger values of the streamwise component of the Reynolds stresses being observed on the left hand side of each image(direction of the incoming crosswind), while the distribution of the spanwise and the vertical components of the stresses are similar to that observed for $\beta=0^{\circ}$. Figure $15 \mathrm{~g}-\mathrm{i}$ show the distribution of the Reynolds normal stresses when the incoming flow is yawed at $\beta=10^{\circ}$. The maximum distribution of the streamwise Reynolds normal stresses $\left(\left\langle u_{x}^{\prime 2}\right\rangle\right)$ occurs in the lower and the side shear layers, stretching from the base of the model and following the curvature of the bounding streamline. The distribution of the spanwise and vertical Reynolds normal stresses are predominantly within the region bounded by the streamwise Reynolds normal stresses, and are nearly symmetrical about the lateral midplane and extends approximately $70 \%-80 \%$ of the height of the GTS model, as opposed to the $\beta=0^{\circ}$, where they are concentrated in the region around the saddle point.

Figure 16 shows the translucent isosurfaces of $\lambda_{2}$ [26] behind the base of the GTS model at the specified yaw angles constructed from the time-averaged velocities coloured by the contours of the pressure coefficient $\left(C_{p}\right)$. For $\beta=2.5^{\circ}$, the change in the flow topology is minimal as compared to $\beta=0^{\circ}$. From these images, a large amount of the vorticity is concentrated along shear layers on the sides of the GTS model and along the bottom where the smaller vortex is observed. This forms a U-shaped structure when viewed from behind the model with a much smaller region of vorticity concentrated in the upper shear layer. The vortex structure in the near wake for $\beta=2.5^{\circ}$ is similar to the non-yawed case. Also visible in Fig. 16a and $\mathrm{b}$ are the corner vortices which originate at the nose of the model and traverse downstream. While these corner vortices are parallel to the sides of the GTS model in Fig. 16a, a small increase in the yaw angle results in these corner vortices shifting away from the incoming flow. While the upper left vortex is shifted rightwards as seen in Fig. 16b; the upper right vortex is not only shifted towards the right, but is also displaced downwards as compared to the non-yawed case (also see Fig. 3 of [70]). The bottom right corner vortex moves upwards and away from the model as it traverses downstream. Also discernible is the separation region at the front of the model which grows larger with increasing yaw angle. At $\beta=10^{\circ}$, the flow structures are more complex than at smaller yaw angles. The corner vortices on the right side of the model are larger for $\beta=10^{\circ}$ as compared to its counterparts at smaller yaw angles. These corner vortices are displaced outwards, away from the model and downwards, as seen in Fig. 16c - e; the upper corner vortex occurs at a location below the height of the model as it approaches a downstream location close the base of the model (also see Fig. 14c). This vortex continues to move closer to the ground as it traverses further downstream, and at a location approximately $2 H$ downstream, this vortex begins to rise vertically upwards as it merges with the corner vortex from the left side of the GTS. The merged vortex is larger and persists at large distances downstream of the base of the model. The flow behaviour observed here is similar to that observed in the wake of other slender bodies such as articulated tractor-trailer models [46, 61] and high-speed trains $[24,29,48]$.

To further examine the flow behaviour when the incoming flow is yawed at $\beta=10^{\circ}$, the contours of the streamwise vorticity are visualised in Fig. 17 at the specified streamwise locations in Fig. 17a, where the contours of pressure coefficient are shown. As the flow is yawed with respect to the vertical midplane of the GTS, a stagnation point on the left side of the GTS model is observed at a height of $\simeq 0.3 \mathrm{H}$ as seen in Fig. $17 \mathrm{~b}$ and c. As the flow separates over the top left corner, three vortices are observed; two smaller vortices 


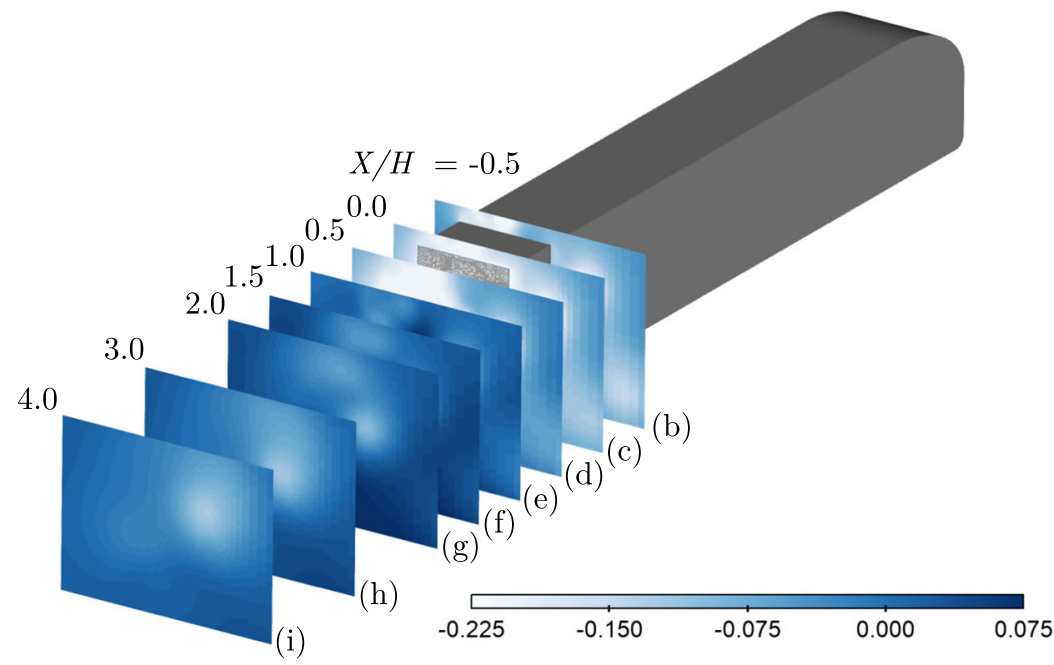

(a)

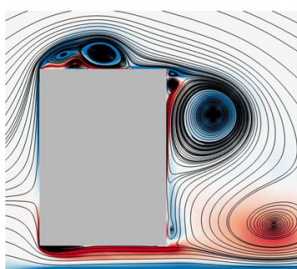

(b)

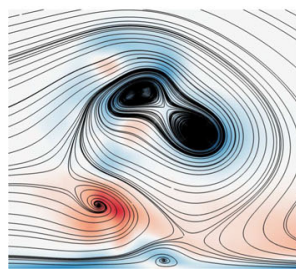

(f)

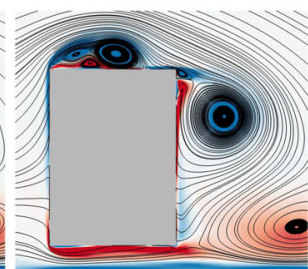

(c)

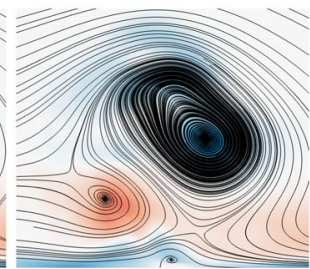

(g)

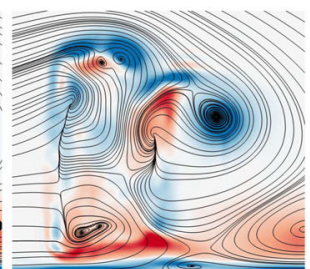

(d)

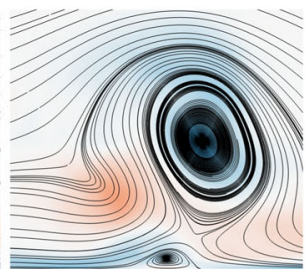

(h)

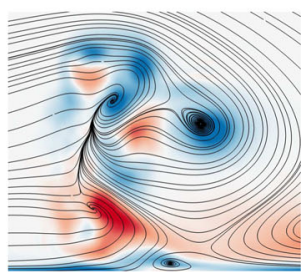

(e)

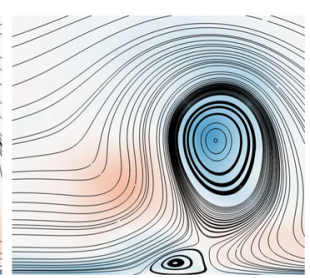

(i)

Fig. 17 a Visualisation of the contours of the pressure coefficient at the specified streamwise locations for the GTS model for the incoming flow at a yaw angle of $\beta=10^{\circ}$. Flow is from top right to bottom left in this image. Contours of the time-averaged streamwise vorticity overlaid with streamlines in the planes shown above for: $\mathbf{b} X / H=-0.5, \mathbf{c} X / H=0 \mathbf{d} X / H=0.5$, e $X / H=1, \mathbf{f} X / H=1.5, \mathbf{g} X / H=2, \mathbf{h} X / H=3$ and $\mathbf{i} X / H=4$. The viewing angle is directly behind the GTS model looking upstream. Contour shading as per Fig. 7

which rotate in the clockwise direction (coloured blue), and one in the anti-clockwise direction (coloured red) closer to the roof of the GTS and in-between the two clockwise rotating vortices. On the right side of the GTS, a larger clockwise vortex is observed, which moves lower and outwards as it moves further downstream (also see Fig. 40 of [65]). Also seen in Fig. 17b and c are smaller vortices formed near the top right corner of the GTS and a smaller vortex at bottom along the edge. These smaller vortices do not extend further downstream and merge into the near-wake. For $X / H \gtrsim 1$, the large vortex on the right hand side combines with the merged vortices from the roof-top and the side (Fig. 17e - g) to form 
Table 2 Comparison of the time-averaged force coefficients on the GTS model for the various cases investigated in this study

\begin{tabular}{lcccr}
\hline Description & Mesh size $(\mathrm{M})$ & $\left\langle C_{x}\right\rangle$ & $C_{y} R M S$ & $\left\langle C_{z}\right\rangle$ \\
\hline Coarse & 3.88 & 0.5724 & 0.0121 & -0.1885 \\
Medium & 8.35 & 0.5734 & 0.0123 & -0.2010 \\
Fine & 10.78 & 0.5608 & 0.0119 & -0.1974 \\
$G / H=1.1$ & 9.18 & 0.5776 & 0.0106 & -0.0588 \\
$\beta=2.5^{\circ}$ & 8.35 & 0.5846 & $0.2923^{*}$ & -0.1822 \\
$\beta=10^{\circ}$ & 8.35 & 0.6803 & $1.3672^{*}$ & 0.0793 \\
$\beta=10^{\circ}$ & 10.78 & 0.6606 & $1.3636^{*}$ & 0.0992 \\
LES $-98 \%$ CDS & 8.35 & 0.5878 & 0.0104 & -0.1994 \\
LES $-100 \%$ CDS & 8.35 & 0.6280 & 0.0107 & -0.1945 \\
\hline
\end{tabular}

The size of the domain in millions of hexahedral elements (M) is also shown. *Note that for the GTS model with the incoming flow at an yaw angle, the mean force coefficient in the spanwise direction $-\left\langle C_{y}\right\rangle$ are presented, while the root-mean-square (RMS) - $C_{y} R M S$ values are shown for the remaining cases. CDS Central differencing scheme in percentage blend

a larger vortex whose height is approximately equal to that of the GTS. This can also be observed in Fig. 17a for $2 \leqslant X / H \leqslant 4$. Also observed in Fig. 17b - e is the bottom corner vortex on the right side of the image which moves further away from the body as it moves downstream. Another vortex which is oppositely signed to the two merging vortices and occurs closer to the ground is observed approximately up to a distance $2 \mathrm{H}$ downstream of the base (Fig. 17e $-\mathrm{g}$ ). However, this vortex is not observed beyond $X / H=3$, while the larger merged clockwise-rotating vortex is the predominant flow structure at large downstream distances, and is accompanied by a smaller vortex close to the ground (Fig. 17h - i).

\subsection{Variation of the force coefficients}

The time-averaged forces experienced on the GTS model for the various configurations tested are detailed in Table 2. Here, $\left\langle C_{x}\right\rangle$ and $\left\langle C_{z}\right\rangle$ correspond to the time-averaged drag and lift force coefficients, respectively, while $C_{y} R M S$ corresponds to the root-mean-square of the side force coefficient. The drag force coefficient experienced by the model with the medium mesh is within $2.5 \%$ of the fine mesh, while the lift coefficient is more sensitive to the increase in resolution. When the incoming flow is yawed by $\beta=2.5^{\circ}$, an increase in the drag coefficient was observed along with a non-zero value of the sideways coefficient. Much larger values of the drag and side force coefficient are observed at larger yaw angles as a result of the larger pressure difference across the sides of the model and the complex flow behaviour in the wake. At a gap height of $1.1 \mathrm{H}$, the drag coefficient is similar to that observed on the medium mesh, indicating the invariance of the drag coefficient for $G / H>$ 0.14 . Despite the large downwash in the anti-symmetric state observed here, the mean lift coefficient has a negative value. The lift coefficient is significantly higher at $G / H=1.1$ as compared to $G / H=0.14$, due to the relatively higher values of pressure coefficient on the underbody of the GTS at the front of the model as compared to the GTS model near the ground. Also shown in Table 2 are the values of the force coefficients with a larger blend of central differencing, where a difference of $\simeq 8.6 \%$ is observed in the values of the drag coefficient between pure central differencing and a blend of $95 \%$ central differencing and $5 \%$ upwind scheme on the medium mesh. 


\section{Conclusions}

An LES study was undertaken to investigate the initial flow topology in the wake of a GTS model at two gap heights of $0.14 \mathrm{H}$ and $1.1 \mathrm{H}$. A spatial resolution study was carried out on three grids, and in all cases, the flow topology in the vertical midplane was found to be antisymmetric to that reported in the experimental works of [45] and [71]. The location of the vortex centres was in good agreement with their experimental counterparts when transposed, thereby, confirming the occurrence of the other bi-stable state in the current simulations. While the phenomenon of bi-stability has previously not been reported in previous experimental studies of the GTS model, the flow is perhaps more averse to switching as compared to that observed in the lateral midplane of the Ahmed body. Furthermore, the underbody support structures used in the experimental investigations may allow the possibility of occurrence of only one of the two flow states reported in the experimental investigations of [45] and [71]. Nonetheless, the run times required to investigate bi-stability numerically would require several hundred flow passages through the domain, and this is computationally prohibitive. When the distance between the ground and the model was increased to $1.1 \mathrm{H}$, the flow topology in the vertical midplane remained unaltered, indicating that the gap height or the underbody flow does not play a significant role in determining the flow topology for $G / H \geqslant 0.14[20,21,45]$.

The influence of crosswinds was also investigated at yaw angles of $\beta=2.5^{\circ}$ and $\beta=$ $10^{\circ}$. For $\beta=2.5^{\circ}$, the flow topology in the vertical midplane remains unaltered as compared to the zero yaw angle case, although an asymmetry is observed in the lateral midplane as a result of the incoming flow at an yaw angle, indicating that the flow topology in the vertical midplane remains unaltered for small yaw angles, as compared to that behind the squareback Ahmed body [76], where a small amount of yawing brought about a change in the flow state. At a larger yaw angle of $\beta=10^{\circ}$, the flow topology is complex, with the merging of the top corner vortices in the far wake. Yawing of the incoming flow with respect to the longitudinal midplane of the GTS brings about a large change in the pressure coefficient at the base of the model, with regions of low pressure developing on the side away from the direction of the incoming crosswind, resulting in a large increase in the drag and side force coefficient.

Thus, the flow topology in the near wake of the GTS model observed in the LES simulations here is invariant over a large range of Reynolds numbers (with a similar flow topology being observed in the vertical midplane as compared to that observed by [49]), and this topology also remains unchanged to an increase in the gap height for $G / H \gtrsim 0.14$, which is in agreement with the findings of [45]. Furthermore, for small values of yaw angle, the initial flow state in the vertical midplane is identical to that at $\beta=0^{\circ}$, indicating that the flow behind the simplified GTS model is rather robust to small changes in the flow configuration. The focus of the current study is primarily to determine the flow topology for a range of configurations, and also to establish a baseline for further investigations using non-zonal hybrid RANS-LES methods such as partially-averaged Navier-Stokes (PANS) equations to accurately predict the near-wake topology on coarser grids [7, 36], which would then result in a reduction of the computational resources required for such simulations.

Acknowledgements The authors would like to thank the computational support provided by Chalmers Centre for Computational Science and Engineering (C3SE) and National Supercomputer Centre (NSC), Linköping University provided by the Swedish National Infrastructure for Computing (SNIC). The authors would also like to acknowledge the support and licences provided by AVL GmbH, Austria. 


\section{Compliance with Ethical Standards}

Conflict of interests The authors declare that they have no conflict of interest.

Open Access This article is distributed under the terms of the Creative Commons Attribution 4.0 International License (http://creativecommons.org/licenses/by/4.0/), which permits unrestricted use, distribution, and reproduction in any medium, provided you give appropriate credit to the original author(s) and the source, provide a link to the Creative Commons license, and indicate if changes were made.

Publisher's Note Springer Nature remains neutral with regard to jurisdictional claims in published maps and institutional affiliations.

\section{References}

1. AVL: FIRE CFD solver users guide. AVL GMbH, Graz (2014)

2. Barros, D., Borée, J., Cadot, O., Spohn, A., Noack, B.R.: Forcing symmetry exchanges and flow reversals in turbulent wakes. J. Fluid Mech. 829. https://doi.org/10.1017/jfm.2017.590 (2017)

3. Bayraktar, I., Landman, D., Baysal, O.: Experimental and computational investigation of Ahmed body for ground vehicle aerodynamics. SAE Technical Paper, SAE International. https://doi.org/10.4271/200101-2742 (2001)

4. Burton, D., McArthur, D., Sheridan, J., Thompson, M.C.: Contribution of add-on components to the aerodynamic drag of a cab-over truck-trailer combination vehicle. SAE Int. J. Commer. Veh. 6, 477-485 (2013). https://doi.org/10.4271/2013-01-2428

5. Cadot, O., Evrard, A., Pastur, L.: Imperfect supercritical bifurcation in a three-dimensional turbulent wake. Phys. Rev. E. 91, 063005 (2015). https://doi.org/10.1103/PhysRevE.91.063005

6. Castelain, T., Michard, M., Szmigiel, M., Chacaton, D., Juvé, D.: Identification of flow classes in the wake of a simplified truck model depending on the underbody velocity. J. Wind Eng. Ind. Aerodyn. 175, 352-363 (2018). https://doi.org/10.1016/j.jweia.2018.02.004. http://www.sciencedirect.com/ science/article/pii/S0167610517307080

7. Chaouat, B.: The state of the art of hybrid RANS/LES modeling for the simulation of turbulent flows. Flow, Turbulence and Combustion 99(2), 279-327 (2017). https://doi.org/10.1007/s10494-017-9828-8

8. Coon, J.D., Visser, K.D.: Drag reduction of a tractor-trailer using planar boat tail plates. In: The Aerodynamics of Heavy Vehicles: Trucks, Buses, and Trains, pp. 249-265. Springer, Berlin (2004)

9. Cooper, K.R.: Wind tunnel and track tests of Class 8 tractors pulling single and tandem trailers fitted with side skirts and boat-tails. SAE Int. J. Commer. Veh. 5, 1-17 (2012). https://doi.org/10.4271/201201-0104

10. Croll, R.H., Gutierrez, W.T., Hassan, B., Suazo, J.E., Riggins, A.J.: Experimental investigation of the ground transportation systems (GTS) project for heavy vehicle drag reduction. SAE technical paper, SAE International. https://doi.org/10.4271/960907 (1996)

11. Das, P., Tsubokura, M., Matsuuki, T., Oshima, N., Kitoh, K.: Large eddy simulation of the flow-field around a full-scale heavy-duty truck. Procedia Engineering 56, 521-530 (2013). https://doi.org/10. 1016/j.proeng.2013.03.155 http://www.sciencedirect.com/science/article/pii/S1877705813005092, 5th BSME International Conference on Thermal Engineering

12. Devesa, A., Indinger, T.: Fuel consumption reduction by geometry variations on a generic tractor-trailer configuration. SAE Int. J. Commer. Veh. 5(1), 18-28 (2012)

13. Duell, E.G., George, A.R.: Experimental study of a ground vehicle body unsteady near wake. SAE Technical Paper, SAE International. https://doi.org/10.4271/1999-01-0812 (1999)

14. El-Alti, M., Chernoray, V., Kjellgren, P., Hjelm, L., Davidson, L.: Computations and full-scale tests of active flow control applied on a VOLVO truck-trailer, pp. 253-267. Springer International Publishing, Cham (2016). https://doi.org/10.1007/978-3-319-20122-1_16

15. Eulalie, Y., Gilotte, P., Mortazavi, I.: Numerical study of flow control strategies for a simplified square back ground vehicle. Fluid Dynamics Research 49(3), 035502 (2017). http://stacks.iop.org/1873-7005/ 49/i=3/a=035502

16. Evrard, A., Cadot, O., Herbert, V., Ricot, D., Vigneron, R., Délery, J.: Fluid force and symmetry breaking modes of a 3D bluff body with a base cavity. J. Fluids Struct. 61, 99-114 (2016). https://doi.org/10.1016/j.jfluidstructs.2015.12.001. http://www.sciencedirect.com/science/article/pii/S0 889974615002698 
17. Ghias, R., Khondge, A., Sovani, S.D.: Flow simulations around a generic ground transportation system: Using immersed boundary method. In: SAE technical paper, SAE International. https://doi.org/10. 4271/2008-01-2613 (2008)

18. Ghosal, S., Moin, P.: The basic equations for the large eddy simulation of turbulent flows in complex geometry. J. Comput. Phys. 118(1), 24-37 (1995)

19. Grandemange, M., Cadot, O., Gohlke, M.: Reflectional symmetry breaking of the separated flow over three-dimensional bluff bodies. Phys. Rev. E. 86, 035,302 (2012). https://doi.org/10.1103/PhysRevE.86. 035302

20. Grandemange, M., Gohlke, M., Cadot, O.: Bi-stability in the turbulent wake past parallelepiped bodies with various aspect ratios and wall effects. Phys. Fluids 25(9), 095103 (2013). https://doi.org/10.1063/1.4820372

21. Grandemange, M., Gohlke, M., Cadot, O.: Turbulent wake past a three-dimensional blunt body. Part 1. Global modes and bi-stability. J. Fluid Mech. 722, 51-84 (2013). https://doi.org/10.1017/jfm.2013.83

22. Grandemange, M., Gohlke, M., Cadot, O.: Turbulent wake past a three-dimensional blunt body. Part 2. Experimental sensitivity analysis. J. Fluid Mech. 752, 439-461 (2014). https://doi.org/10.1017/jfm. 2014.345

23. Gunes, D.: On the similarity of wind tunnel experiments and numerical simulation of heavy-duty trailer flow. Progress in Computational Fluid Dynamics, an International Journal 10(3), 168-176 (2010). https://doi.org/10.1504/PCFD.2010.033328

24. Hemida, H., Krajnović, S.: LES study of the influence of the nose shape and yaw angles on flow structures around trains. J. Wind Eng. Ind. Aerodyn. 98(1), 34-46 (2010). https://doi.org/10.1016/j.jweia. 2009.08.012 http://www.sciencedirect.com/science/article/pii/S0167610509000865

25. Hunt, J.C.R., Abell, C.J., Peterka, J.A., Woo, H.: Kinematical studies of the flows around free or surfacemounted obstacles; applying topology to flow visualization. J. Fluid Mech. 86(1), 179-200 (1978). https://doi.org/10.1017/S0022112078001068

26. Jeong, J., Hussain, F.: On the identification of a vortex. J. Fluid Mech. 285, 69-94 (1995)

27. Khalighi, B., Chen, K.H., Iaccarino, G.: Unsteady aerodynamic flow investigation around a simplified square-back road vehicle with drag reduction devices. J. Fluids Eng. 134(6), 061,101-061,101-16 (2012). https://doi.org/10.1115/1.4006643

28. Kim, J.J., Lee, S., Kim, M., You, D., Lee, S.J.: Salient drag reduction of a heavy vehicle using modified cab-roof fairings. J. Wind Eng. Ind. Aerodyn. 164, 138-151 (2017). https://doi.org/10.1016/j.jweia.2017. 02.015 http://www.sciencedirect.com/science/article/pii/S0167610516301337

29. Krajnović, S.: Large eddy simulation of flows around ground vehicles and other bluff bodies. Philosophical Transactions of the Royal Society of London A: Mathematical, Physical and Engineering Sciences 367(1899), 2917-2930 (2009). https://doi.org/10.1098/rsta.2009.0021

30. Krajnović, S.: Large eddy simulation exploration of passive flow control around an Ahmed body. J. Fluids Eng. 136(12), 121,103-121,103-10 (2014). https://doi.org/10.1115/1.4027221

31. Krajnović, S.: What can LES do in vehicle aerodynamics? pp. 311-326. Springer International Publishing, Berlin (2016)

32. Krajnović, S., Davidson, L.: Large-eddy simulation of the flow around a bluff body. AIAA J. 40(5), 927-936 (2002). https://doi.org/10.2514/2.1729

33. Krajnović, S., Davidson, L.: Numerical study of the flow around a bus-shaped body. J. Fluids Eng. 125(3), 500-509 (2003). https://doi.org/10.1115/1.1567305

34. Krajnović, S., Davidson, L.: Exploring the flow around a simplified bus with large eddy simulation and topological tools. In: The aerodynamics of heavy vehicles: trucks, buses, and trains, pp. 49-64. Springer, Berlin (2004)

35. Krajnović, S., Davidson, L.: Large eddy simulation of the flow around an Ahmed body. https://doi.org/10.1115/HT-FED2004-56325 (2004)

36. Krajnović, S., Lárusson, R., Basara, B.: Superiority of PANS compared to LES in predicting a rudimentary landing gear flow with affordable meshes. Int. J. Heat Fluid Flow 37, 109-122 (2012). https://doi. org/10.1016/j.ijheatfluidflow.2012.04.013

37. Krajnović, S., Minelli, G., Basara, B.: Partially-Averaged Navier-Stokes simulations of two bluff body flows. Appl. Math. Comput. 272(Part 3), 692-706 (2016)

38. Lahaye, A., Leroy, A., Kourta, A.: Characterisation of a square back Ahmed body near wake flow. In: 21 ème Congrés Français De Mécanqiue, Bordeaux (2013)

39. Lahaye, A., Leroy, A., Kourta, A.: Aerodynamic characterisation of a square back bluff body flow. Int. J. Aerodyn. 4(1-2), 43-60 (2014). https://doi.org/10.1504/IJAD.2014.057804

40. Larsson, T., Hammara, J., Barthb, M.L.A.: Scale resolving CFD and CAA processes for ground vehicles based on Open Source. available online pp. 1-13. http://www.prace-ri.eu/IMG/pdf/WP260.pdf (2017) 
41. Lu, F.: Surface oil flow visualization. The European Physical Journal Special Topics 182(1), 51-63 (2010)

42. Lucas, J.M., Cadot, O., Herbert, V., Parpais, S., Délery, J.: A numerical investigation of the asymmetric wake mode of a squareback Ahmed body - effect of a base cavity. J. Fluid Mech. 831, 675-697 (2017). https://doi.org/10.1017/jfm.2017.654

43. Maddox, S., Squires, K.D., Wurtzler, K.E., Forsythe, J.R.: Detached-eddy simulation of the ground transportation system. In: The aerodynamics of heavy vehicles: trucks, buses, and trains, pp. 89-104. Springer, Berlin (2004). https://doi.org/10.1007/978-3-540-44419-0_8

44. Martini, H., Bergqvist, B., Hjelm, L., Löfdahl, L.: Aerodynamic Effects of Roof Deflector and Cab Side Extenders for Truck-Trailer Combinations. SAE Technical Paper, SAE International (2011)

45. McArthur, D., Burton, D., Thompson, M.C., Sheridan, J.: On the near wake of a simplified heavy vehicle. J. Fluids Struct. 66, 293-314 (2016). https://doi.org/10.1016/j.jfluidstructs.2016.07.011. http://www. sciencedirect.com/science/article/pii/S0889974615301043

46. McArthur, D., Burton, D., Thompson, M.C., Sheridan, J.: An experimental characterisation of the wake of a detailed heavy vehicle in cross-wind. J. Wind Eng. Ind. Aerodyn. 175, 364 375 (2018). https://doi.org/10.1016/j.jweia.2018.01.033. http://www.sciencedirect.com/science/article/ pii/S0167610517304737

47. Minelli, G., Krajnović, S., Basara, B., Noack, B.R.: Numerical investigation of active flow control around a generic truck A-pillar. Flow, Turbulence and Combustion 97(4), 1235-1254 (2016)

48. Munoz-Paniagua, J., García, J., Lehugeur, B.: Evaluation of RANS, SAS and IDDES models for the simulation of the flow around a high-speed train subjected to crosswind. J. Wind Eng. Ind. Aerodyn. 171, 50-66 (2017). https://doi.org/10.1016/j.jweia.2017.09.006. http://www.sciencedirect.com/science/ article/pii/S0167610516306924

49. Ortega, J.M., Dunn, T., McCallen, R., Salari, K.: Computational simulation of a heavy vehicle trailer wake. In: The aerodynamics of heavy vehicles: trucks, buses, and trains, pp. 219-233. Springer, Berlin (2004). https://doi.org/10.1007/978-3-540-44419-0_22

50. Östh, J., Krajnović, S.: A study of the aerodynamics of a generic container freight wagon using LargeEddy Simulation. J. Fluids Struct. 44, 31-51 (2014)

51. Östh, J., Krajnović, S.: A LES study of a simplified tractor-trailer model. The Aerodynamics of Heavy Vehicles III, Springer, pp. 327-342 (2016)

52. Östh, J., Krajnović, S., Basara, B.: LES study of breakdown control of A-pillar vortex. International Journal of Flow Control 2(4), 237-258 (2010)

53. Östh, J., Noack, B., Krajnović, S., Barros, D., Borée, J.: On the need for a nonlinear subscale turbulence term in POD models as exemplified for a high-Reynolds-number flow over an Ahmed body. J. Fluid Mech. 747, 518-544 (2014)

54. Pankajakshan, R., Hilbert, C.B., Whitfield, D.L.: Passive Devices for Reducing Base Pressure Drag in Class 8 Trucks. In: Dillmann, A., Orellano, A. (eds.) The Aerodynamics of Heavy Vehicles III, pp. 227 235. Springer International Publishing, Cham (2016)

55. Parkin, D., Sheridan, J., Thompson, M.C.: Numerical analysis of periodic open-loop flow control on bluff bodies in ground proximity. J. Wind Eng. Ind. Aerodyn. 145, 339-350 (2015). https://doi.org/10.1016/ j.jweia.2015.03.016 http://www.sciencedirect.com/science/article/pii/S0167610515000768

56. Parkin, D.J., Thompson, M.C., Sheridan, J.: Numerical analysis of bluff body wakes under periodic open-loop control. J. Fluid Mech. 739, 94-123 (2014). https://doi.org/10.1017/jfm.2013.618

57. Patankar, S.V., Spalding, D.: A calculation procedure for heat, mass and momentum transfer in threedimensional parabolic flows. International Journal of Heat and Mass Transfer 15(10), 1787-1806 (1972)

58. Pavia, G., Passmore, M., Sardu, C.: Evolution of the bi-stable wake of a square-back automotive shape. Exp. Fluids 59(1), 20 (2017)

59. Perry, A.K., Pavia, G., Passmore, M.: Influence of short rear end tapers on the wake of a simplified square-back vehicle: wake topology and rear drag. Exp. Fluids 57(11), 169 (2016)

60. Piomelli, U., Chasnov, J.R.: Large-Eddy Simulations: Theory and applications, pp. 269-336. Springer, Netherlands (1996)

61. Pointer, W.D., Sofu, T., Weber, D.: Development of guidelines for the use of commercial CFD in tractortrailer aerodynamic design. 2005 SAE Commercial vehicle engineering conference, SAE International. https://doi.org/10.4271/2005-01-3513 (2005)

62. Pope, S.B.: Turbulent Flows. Cambridge University Press, Cambridge (2000). https://doi.org/10.1017/ CBO9780511840531

63. Roy, C., Payne, J., McWherter-Payne, M.: RANS simulations of a simplified tractor/trailer geometry. J. Fluids Eng. 128(5), 1083-1089 (2006). https://doi.org/10.1115/1.2236133

64. Sagaut, P.: Large Eddy Simulation for Incompressible Flows: An Introduction Scientific Computation. Springer, Berlin (2006). https://link.springer.com/book/10.1007/b137536 
65. Salari, K., Ortega, J., Castellucci, P.: Computational prediction of aerodynamic forces for a simplified integrated tractor-trailer geometry. 34th AIAA Fluid Dynamics Conference and Exhibit, Fluid Dynamics and Co-located Conferences, American Institute of Aeronautics and Astronautics, pp 1-53. https://doi.org/10.2514/6.2004-2253 (2004)

66. Schlichting, H., Gersten, K. Boundary-Layer Theory, 9th. Springer, Berlin (2017)

67. Schmidt, H.-J., Woszidlo, R., Nayeri, C.N., Paschereit, C.O.: The effect of flow control on the wake dynamics of a rectangular bluff body in ground proximity. Exp. Fluids 59(6), 107 (2018). https://doi.org/10.1007/s00348-018-2560-x. ISSN 1432-1114

68. Smagorinsky, J.: General Circulation Experiments with the Primitive Equations. Mon. Weather Rev. 91, 99 (1963). https://doi.org/10.1175/1520-0493(1963)091 〈0099:GCEWTP $\rangle$ 2.3.CO;2

69. Sohankar, A., Davidson, L., Norberg, C.: Large eddy simulation of flow past a square cylinder: Comparison of different subgrid scale models. J. Fluids Eng. 122(1), 39-47 (1999). https://doi.org/10.1115/1.483 224

70. Spalart, P.R., Squires, K.D.: The status of detached-eddy simulation for bluff bodies. In: The aerodynamics of heavy vehicles: trucks, buses, and trains, pp. 29-45. Springer, Berlin (2004). https://doi.org/10. 1007/978-3-540-44419-0_3

71. Storms, B., Ross, J., Heineck, J., Walker, S., Driver, D., Zilliac, G.: An experimental study of the ground transportation system (GTS) model in the NASA Ames 7-by 10-ft wind tunnel. National Aeronautics and Space Administration Ames Research Center, pp. 1-21 (2001)

72. Sujudi, D., Haimes, R.: Identification of Swirling Flow in 3-D Vector Fields. In: 12th Computational Fluid Dynamics Conference Co-located Conferences. American Institute of Aeronautics and Astronautics, San Diego (1995)

73. Unaune, S.V., Sovani, S.D., Kim, S.E.: Aerodynamics of a generic ground transportation system: Detached eddy simulation. In: SAE Technical Paper, SAE International. https://doi.org/10.4271/200501-0548 (2005)

74. Van Raemdonck, G.M.R.: Design of Low Drag Bluff Road Vehicles PhD Dissertation. TU Delft, Delft University of Technology (2012)

75. Varon, E., Eulalie, Y., Edwige, S., Gilotte, P., Aider, J.L.: Control of the chaotic dynamics of a turbulent 3D wake. available online arXiv:1712.00416 (2017)

76. Volpe, R., Devinant, P., Kourta, A.: Unsteady experimental characterization of the natural wake of a squareback Ahmed model. ASME $20144^{\text {th }}$ Joint US-European Fluids Engineering Division Summer Meeting collocated with the ASME 2014 12th International Conference on Nanochannels, Microchannels, and Minichannels, ASME, 46230, pp. V01CT17A007-1-V01CT17A007-7. https://doi.org/10.1115/FEDSM2014-21494 (2014)

77. Volpe, R., Ferrand, V., Silva, A.D., Moyne, L.L.: Forces and flow structures evolution on a car body in a sudden crosswind. J. Wind Eng. Ind. Aerodyn. 128, 114-125 (2014). http://oatao.univ-toulouse.fr/ $11870 /$

78. Volpe, R., Devinant, P., Kourta, A.: Experimental characterization of the unsteady natural wake of the full-scale square back Ahmed body: flow bi-stability and spectral analysis. Exp. Fluids 56, 99 (2015). https://doi.org/10.1007/s00348-015-1972-0

79. Winkler, N., Drugge, L., Trigell, A.S., Efraimsson, G.: Coupling aerodynamics to vehicle dynamics in transient crosswinds including a driver model. Comput. Fluids 138(Supplement C), 26-34 (2016). https://doi.org/10.1016/j.compfluid.2016.08.006. http://www.sciencedirect.com/science/article/ pii/S004579301630250X 